\title{
Polarization transfer in weak pion production off the nucleon
}

\author{
Krzysztof M. Graczyk* and Beata E. Kowal \\ Institute of Theoretical Physics, University of Wrocław, plac Maxa Borna 9, 50-204 Wrocław, Poland
}

(Received 15 November 2017; published 8 January 2018)

\begin{abstract}
Polarization transfer (PT) observables in the single pion production induced by the charged current interaction of the neutrino with the nucleon are examined. The polarization components of the final nucleon and the charged lepton are calculated within two models for the pion production. The predictions are made for neutrino energy of the order of $1 \mathrm{GeV}$ as well as for the T2K energy distribution. It is demonstrated that the PT observables, the degree of polarization and the polarization components of outgoing fermions, are sensitive to assumptions about the nonresonant background model. In particular it is shown that the normal components of the polarization of the outgoing nucleon and the lepton are determined by the interference between the resonant (RES) and nonresonant (NB) amplitudes. Moreover, the sign of the normal component of the polarization of the charged lepton is fixed by the relative sign between the RES and the NB amplitudes.
\end{abstract}

DOI: $10.1103 /$ PhysRevD.97.013001

\section{INTRODUCTION}

The first theoretical studies of the single pion production (SPP) in neutrino-nucleon $(\nu N)$ scattering were performed more than 40 years ago. A historical review of the topic can be found in [1]. The latest developments of the accelerator neutrino oscillation experiments [2-5] have triggered new interest in SPP processes.

Further progress in the investigation of the fundamental properties of neutrinos (the oscillation phenomenon, the $C P$-violation in the lepton sector, and the mass hierarchy) requires an improvement of the experimental techniques for the measurement of the interactions of neutrinos with different nuclear targets [6] as well as a development of the theoretical models describing the neutrino-nucleus scattering [7].

The measurement of the neutrino oscillation parameters and extraction of the $C P$-violation phase are made based on the analysis of the quasielastic $(\mathrm{QE})$ neutrino-nucleus scattering; however, the SPP processes contribute to the background for the detection of the QE-like events as well as the electron neutrinos in the far detector. Hence the SPP contribution cannot be neglected in the analyses of neutrino scattering data. Additionally, the investigation of the SPP in the $\nu N$ interactions allows us to study the weak excitation of the nucleon to the resonance states.

\footnotetext{
*krzysztof.graczyk@uwr.edu.pl
}

Published by the American Physical Society under the terms of the Creative Commons Attribution 4.0 International license. Further distribution of this work must maintain attribution to the author(s) and the published article's title, journal citation, and DOI. Funded by SCOAP.
A first natural step in modeling the SPP in $\nu$-nucleus scattering is the construction of the theoretical description for the $\nu$-nucleon scattering. In this work we focus on the interactions of neutrinos with a free nucleon target. Two mechanisms for the pion production in the $\nu N$ scattering can be distinguished: a resonant (RES) and a nonresonant mechanism. In the first, the nucleon is excited to the resonance state, $N^{*}$, which subsequently decays to a $\pi N$ system. In the other, there is no $N \rightarrow N^{*}$ transition. This contribution can be modeled by the so-called nonresonant background (NB) amplitudes allowed by the symmetries [8].

The choice of the degrees of freedom of the SPP model depends on the energy range in which it is applicable. If neutrino energy is relatively low, $E \sim 1 \mathrm{GeV}$, it is enough to consider the contribution to the scattering amplitude from the first resonance region. For larger energies one has to include also the resonance states from the second and third resonance regions, as well as higher order NB terms. In this work we discuss neutrino energy of the order of $1 \mathrm{GeV}$, which is a kinematic domain typical for the long and short baseline experiments with an accelerator source of neutrinos $[2,3]$.

There are many phenomenological models describing the SPP in $\nu N$ interactions [9-20]; for a more complete list, see $[7,21]$. One of the main difficulties in modeling the pion production is proposing a consistent description of both the RES and the NB contributions. The analysis of the unpolarized cross section data does not allow us to distinguish between the RES and NB contributions. Moreover, the main information about the SPP in $\nu N$ scattering is obtained from the analysis of the data collected by two bubble chamber experiments Argonne National 
Laboratory (ANL) [22] and Brookhaven National Laboratory (BNL) [23], in which the neutrino-deuteron scattering was investigated. There are new measurements of the SPP in the $\nu$-nucleus scattering, e.g., by Minerva [24] experiment. But in the analysis of these data, the nuclear structure effects must be included, which makes the studies complicated and model dependent.

In this work we propose to study the polarization properties of the particles in the SPP processes. We shall show that the polarization transfer (PT) observables contain the nontrivial information about the resonance and the nonresonace transition. In particular we demonstrate that the investigation of the normal polarization components of the charged lepton and the final nucleon give knowledge about the relations between the RES and NB contributions. Indeed the normal polarizations are proportional to the interference of the RES and NB amplitudes.

The PT observables have been studied experimentally and theoretically in electron scattering off the nucleon/ nuclei in past years. More than 40 years ago, Akhiezer et al. [25,26] (see also [27-29]) showed that from the measurement of the PT observables in the elastic electron scattering off the proton, the form factor ratio $G_{E}^{p} / G_{M}^{p}$ $\left(G_{E / M}^{p}\right.$ is the electric/magnetic proton form factor of the proton) can be obtained. This is an alternative method for the measurement of the elastic form factors to the famous Rosenbluth method. The ratio $G_{E}^{p} / G_{M}^{p}$ obtained from the PT data turned out to be inconsistent with the Rosenbluth measurements. It triggered off more detailed experimental and theoretical studies of the elastic electron-nucleon scattering. A recent review of the topic can be found in [30].

The investigation of the polarization properties of final particles in the $\nu N$ scattering is not a new idea. The PT observables in the $\mathrm{QE} \nu N$ scattering were discussed in [1]. Recently the problem has been refreshed in Refs. [31-33]. Moreover, in Refs. [34-36] the polarization properties of the $\tau$ lepton produced in the $\mathrm{QE}$ and inelastic $\nu N$ interactions were studied. Additionally, in [37] the impact of the nuclear effects on the polarization of the $\tau$ lepton produced in the QE neutrino-nucleus scattering was investigated.

The polarization properties of the $\tau$ lepton in the SPP induced by the $\nu_{\tau} N$ scattering processes were studied by two groups: Hagiwara et al. [35] and Naumov et al. [34]. However, the discussed SPP models did not contain the NB contribution. In our work the NB contribution plays a central role. We show that the PT observables are sensitive to the NB contribution. We discuss the charged current interactions of the muon and the tau neutrinos with the nucleons. Eventually we investigate also the polarization properties of the final nucleon produced in the SPP process. This problem has been not studied before.

We show that the PT observables are sensitive to the various details of the SPP models, in particular the description of the NB contribution. In order to study the model dependence of the predictions of the PT observables, we consider two phenomenological approaches for the SPP: the Hernandez-Nieves-Valverde (HNV) model as formulated in [8] and the Fogli-Nardulli (FN) model as described in [11]. Both approaches are similar in construction but it is demonstrated that small differences in the treatment of the NB contribution give rise to disparities in the predictions of the PT observables.

The paper is organized as follows: Section II introduces kinematics and the cross section formula, in Sec. III the polarization observables are given, Sec. IV contains a short review of the HNV and FN models, and in Sec. V the numerical results are presented and discussed. We summarize in Sec. VI. Additionally, we include three appendixes.

\section{KINEMATICS AND CROSS SECTION}

We consider the SPP induced by the charged current (CC) $\nu N$ interaction

$$
\nu_{l}(k)+N(p) \rightarrow l^{-}\left(k^{\prime}\right)+N^{\prime}\left(p^{\prime}\right)+\pi\left(k_{\pi}\right),
$$

where $l=\mu, \tau ; k^{\alpha}=(E, \mathbf{k})$; and $k^{\prime \alpha}=\left(E^{\prime}, \mathbf{k}^{\prime}\right)$ are the four-momenta of the initial and the final leptons, respectively, while $p^{\alpha}=\left(E_{p}, \mathbf{p}\right) ; \quad p^{\prime \alpha}=\left(E_{p^{\prime}}, \mathbf{p}^{\prime}\right) ;$ and $k_{\pi}^{\alpha}=$ $\left(E_{\pi}, \mathbf{k}_{\pi}\right)$ denote the four-momenta of the incoming nucleon $(\mathrm{N})$, the outgoing nucleon $\left(N^{\prime}\right)$, and the pion, respectively. Notice that $E_{x}=\sqrt{\mathbf{x}^{2}+M_{x}^{2}} \cdot M, m$, and $m_{\pi}$ denote masses of the nucleon, charged lepton, and pion, respectively.

The four-momentum transfer is defined as

$$
q^{\alpha} \equiv k^{\alpha}-k^{\prime \alpha}=(\omega, \mathbf{q}),
$$

and the invariant hadronic mass $W$ is given by

$$
W^{2}=(p+q) \cdot(p+q) \equiv(p+q)_{\mu}(p+q)^{\mu}=(p+q)^{2} .
$$

Let us also define

$$
Q^{2} \equiv-q^{2}
$$

The scattering angle between lepton momenta is denoted $\theta \equiv \angle\left(\mathbf{k}, \mathbf{k}^{\prime}\right)$, while the spherical angle (depending on $\theta$ ) is denoted by $\Omega ; \phi_{\pi}$ is the angle between the scattering plane (spanned by $\mathbf{k}$ and $\mathbf{k}^{\prime}$ ) and the plane spanned by the pion and the final nucleon momenta (see Fig. 1).

The differential cross section for the process (1) reads

$$
\begin{aligned}
d \sigma= & \frac{1}{4 M E_{k}} \sum_{\text {spins spins }} \sum^{\prime} \frac{d^{3} \mathbf{k}^{\prime}}{2 E_{k^{\prime}}(2 \pi)^{3}} \frac{d^{3} \mathbf{p}^{\prime}}{2 E_{p^{\prime}}(2 \pi)^{3}} \frac{d^{3} \mathbf{k}_{\pi}}{2 E_{\pi}(2 \pi)^{3}} \\
& \times(2 \pi)^{4} \delta^{(4)}\left(p^{\prime}+k_{\pi}+k^{\prime}-k-p\right)\left|\mathcal{M}_{f i}\right|^{2} .
\end{aligned}
$$




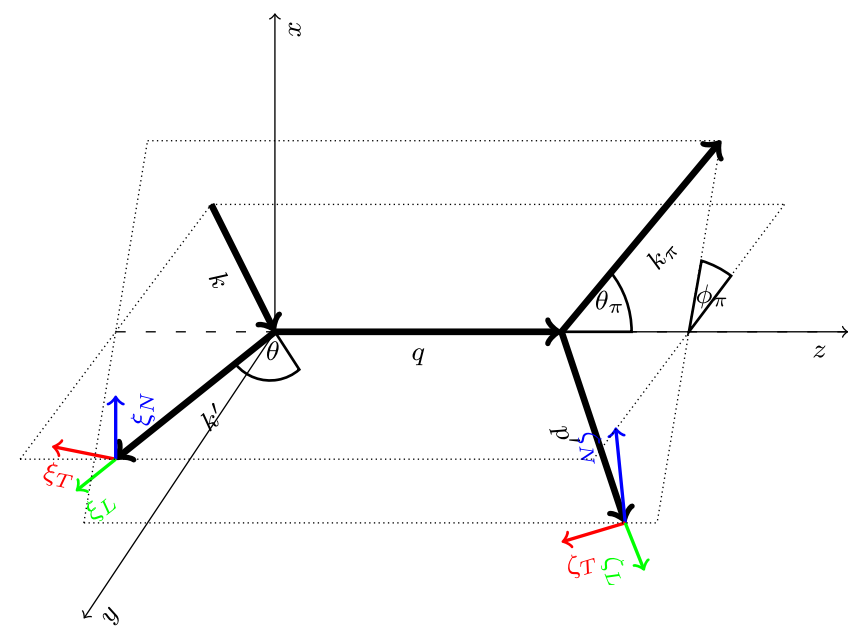

FIG. 1. Angular distribution of the particles, in the laboratory frame, in the process (1). The momenta $k$ and $k^{\prime}$ denote the neutrino and the charged lepton, respectively. The target is at rest, while $k_{\pi}$ and $p^{\prime}$ denote the pion and the outgoing nucleon momenta. The vectors $\xi$ and $\zeta$ in green, blue, and red denote longitudinal, normal, and transverse components of the charged lepton and the nucleon, respectively.

Sum over the spins of the leptons and the nucleons is denoted by $\sum^{\prime}$ and $\sum$, respectively. We systematically omit the spin notation in this section.

The scattering matrix, in the one-boson exchange approximation and for $-q^{2} \ll M_{W}^{2}$, reads

$\mathcal{M}_{f i}=-\frac{G_{F}}{\sqrt{2}} \cos \theta_{C} \bar{u}\left(k^{\prime}\right) \gamma_{\mu}\left(1-\gamma_{5}\right) u(k)\left\langle\pi, N^{\prime}\left|\mathcal{J}^{\mu}\right| N\right\rangle$,

where $G_{F} \approx 1.16639 \times 10^{-5} \mathrm{GeV}^{-2}$ is the Fermi constant, while $\theta_{C}$ is the Cabibbo angle and $\cos \theta_{C} \approx 0.97427$. The expectation value of the CC weak hadronic current operator $\mathcal{J}^{\mu}$ reads

$$
J^{\mu} \equiv\left\langle\pi, N^{\prime}\left|\mathcal{J}^{\mu}\right| N\right\rangle
$$

Distinct phenomenological models can contain different numbers of diagrams contributing to the total scattering amplitude. Hence the total hadronic current can be given by the sum

$$
J^{\mu} \rightarrow \sum_{a \in S} \mathcal{C}^{a} J_{a}^{\mu}
$$

where $\mathcal{C}^{a}$ is the Clebsch-Gordan coefficient; see Table I.

The total amplitude in the HNV model is described by seven Feynman diagrams, hence

$$
\mathcal{S}_{\mathrm{HNV}}=\{\mathrm{NP}, \mathrm{CNP}, \mathrm{CT}, \mathrm{PP}, \mathrm{PF}, \Delta P, C \Delta P\} .
$$

TABLE I. Clebsch-Gordan coefficients.

\begin{tabular}{lccccccc}
\hline \hline Process & $\mathrm{NP}$ & $\mathrm{CNP}$ & $\mathrm{CT}$ & $\mathrm{PP}$ & $\mathrm{PF}$ & $\Delta P$ & $C \Delta P$ \\
\hline$\nu_{l} p \rightarrow l^{-} p \pi^{+}$ & 0 & 1 & 1 & 1 & 1 & 1 & 1 \\
$\nu_{l} n \rightarrow l^{-} p \pi^{0}$ & $\frac{1}{\sqrt{2}}$ & $-\frac{1}{\sqrt{2}}$ & $-\sqrt{2}$ & $-\sqrt{2}$ & $-\sqrt{2}$ & $-\frac{\sqrt{2}}{3}$ & $\sqrt{2}$ \\
$\nu_{l} n \rightarrow l^{-} n \pi^{+}$ & 1 & 0 & -1 & -1 & -1 & $\frac{1}{3}$ & 3 \\
$\bar{\nu}_{l} n \rightarrow l^{+} n \pi^{-}$ & 0 & 1 & 1 & 1 & 1 & 1 & 1 \\
$\bar{\nu}_{l} p \rightarrow l^{+} n \pi^{0}$ & $-\frac{1}{\sqrt{2}}$ & $\frac{1}{\sqrt{2}}$ & $\sqrt{2}$ & $\sqrt{2}$ & $\sqrt{2}$ & $\frac{\sqrt{2}}{3}$ & $-\sqrt{2}$ \\
$\bar{\nu}_{l} p \rightarrow l^{+} p \pi^{-}$ & 1 & 0 & -1 & -1 & -1 & $\frac{1}{3}$ & 3 \\
\hline \hline
\end{tabular}

An explanation of the notation in the parentheses can be found in Fig. 2 and Ref. [8].

In the FN model, the total amplitude is given by four diagrams:

$$
\mathcal{S}_{\mathrm{FN}}=\{\mathrm{NP}, \mathrm{CNP}, \mathrm{PF}, \Delta P\} .
$$

The structure of the amplitudes in the HNV and FN models is the same; hence, we introduce

$$
J_{a}^{\mu}=\left\langle\pi, N^{\prime}\left|\mathcal{J}_{a}^{\mu}(0)\right| N\right\rangle=\bar{u}\left(p^{\prime}\right) R_{a}^{\mu} u(p),
$$

where $R_{a}^{\mu}=r_{a}^{\mu}$ and $R_{a}^{\mu}=\tilde{r}^{\mu}$ for the $\mathrm{HNV}$ and the FN models, respectively.

Eventually it is convenient to introduce the notation

$$
\mathcal{M}_{a}=-\frac{G_{F}}{\sqrt{2}} \cos \theta_{C} \bar{u}\left(k^{\prime}\right) \gamma_{\mu}\left(1-\gamma_{5}\right) u(k) R_{a}^{\mu},
$$

and then

$$
\mathcal{M}_{f i}=\sum_{a \in \mathcal{S}} \mathcal{M}_{a}
$$

\section{POLARIZATION TRANSFER OBSERVABLES}

We consider two types of PT processes. In the first, the polarization of the final lepton is examined, namely,

$$
\nu_{l}(k)+N(p) \rightarrow \vec{l}^{-}\left(k^{\prime}, \xi\right)+N^{\prime}\left(p^{\prime}\right)+\pi\left(k_{\pi}\right) .
$$

In the other, the polarization of the final nucleon is the subject of study:

$$
\nu_{l}(k)+N(p) \rightarrow l^{-}\left(k^{\prime}\right)+\vec{N}^{\prime}\left(p^{\prime}, \zeta\right)+\pi\left(k_{\pi}\right) .
$$

Notice that $\xi$ and $\zeta$ are the four-vector spins of the final lepton and the nucleon, respectively.

A spin four-vector $s^{\mu}$ of a $1 / 2$-spin fermion of mass $M$ has three independent components. At rest it has the form 


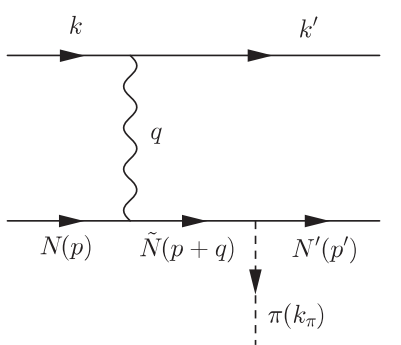

(a)

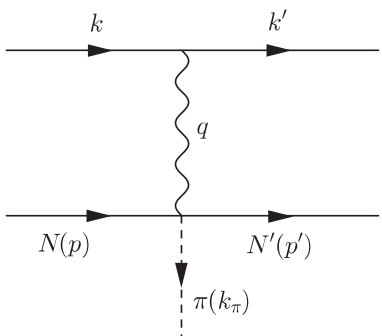

(c)

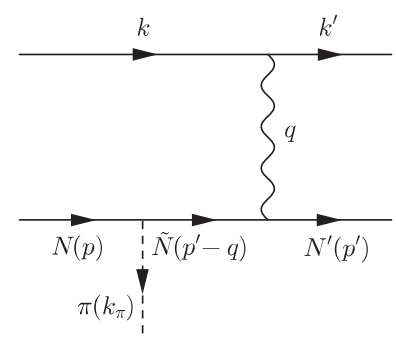

(b)

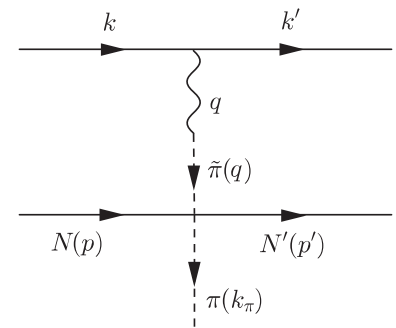

(d)

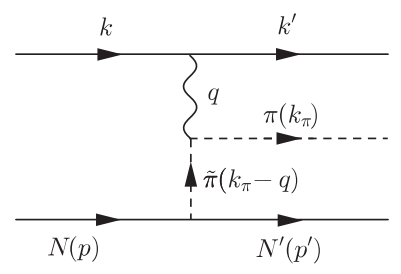

(e)

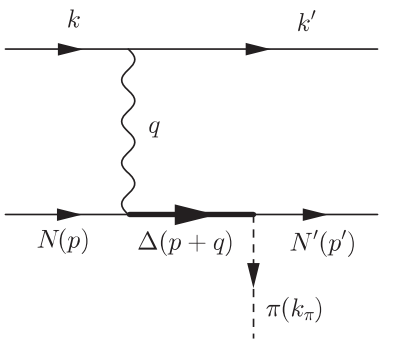

(f)

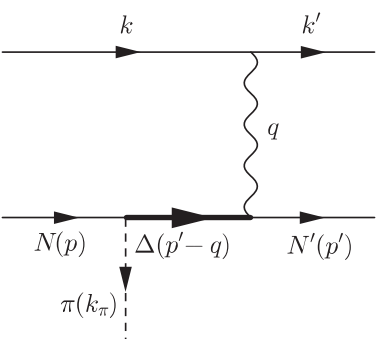

(g)

FIG. 2. Diagrams for the SPP in $\nu N$ scattering. The NB contribution is given by (a) nucleon pole (NP); (b) crossed nucleon pole (CNP); (c) contact term (CT); (d) pion pole (PP); (e) pion in flight (PF). The RES contribution is given by (f) delta pole $(\Delta P) ;(\mathrm{g})$ crossed delta pole $(C \Delta P) . \tilde{N}$ denotes a virtual nucleon.

$$
s^{\mu}=(0, \hat{\mathbf{s}}), \quad \text { where } \hat{\mathbf{s}}^{2}=1
$$

In a frame in which a particle moves with the velocity $\mathbf{p} / E_{p}$, the spin vector reads [38-40]

$$
s^{\mu}=\left(\frac{\hat{\boldsymbol{s}} \cdot \mathbf{p}}{M}, \hat{\boldsymbol{s}}+\mathbf{p} \frac{\hat{\boldsymbol{s}} \cdot \mathbf{p}}{M\left(M+E_{p}\right)}\right) .
$$

In any frame,

$$
s \cdot p=0 \text {. }
$$

It is convenient to introduce longitudinal $(L)$, transverse $(T)$, and normal $(N)$ components of the spin,

$$
\begin{aligned}
& \xi^{\mu}=\xi_{L}^{\mu}+\xi_{T}^{\mu}+\xi_{N}^{\mu}, \\
& \zeta^{\mu}=\zeta_{L}^{\mu}+\zeta_{T}^{\mu}+\zeta_{N}^{\mu},
\end{aligned}
$$

where we choose the coordinates so that (see Fig. 1)

$$
\begin{gathered}
\xi_{L}^{\mu}=h_{l}\left(\frac{\left|\mathbf{k}^{\prime}\right|}{m}, \frac{E_{k^{\prime}}}{m} \frac{\mathbf{k}^{\prime}}{\left|\mathbf{k}^{\prime}\right|}\right), \\
\xi_{N}^{\mu}=h_{l}\left(0, \frac{\mathbf{k} \times \mathbf{q}}{|\mathbf{k} \times \mathbf{q}|}\right),
\end{gathered}
$$

$$
\xi_{T}^{\mu}=h_{l}\left(0, \frac{\mathbf{k}^{\prime} \times(\mathbf{k} \times \mathbf{q})}{\left|\mathbf{k}^{\prime} \times(\mathbf{q} \times \mathbf{k})\right|}\right),
$$

and $h_{l}= \pm 1$ as well as

$$
\begin{gathered}
\zeta_{L}^{\mu}=h_{N}\left(\frac{\left|\mathbf{p}^{\prime}\right|}{M}, \frac{E_{p^{\prime}}}{M} \frac{\mathbf{p}^{\prime}}{\left|\mathbf{p}^{\prime}\right|}\right), \\
\zeta_{N}^{\mu}=h_{N}\left(0, \frac{\mathbf{p}^{\prime} \times \mathbf{k}_{\pi}}{\left|\mathbf{p}^{\prime} \times \mathbf{k}_{\pi}\right|}\right), \\
\zeta_{T}^{\mu}=h_{N}\left(0, \frac{\mathbf{p}^{\prime} \times\left(\mathbf{p}^{\prime} \times \mathbf{k}_{\pi}\right)}{\left|\mathbf{p}^{\prime} \times\left(\mathbf{p}^{\prime} \times \mathbf{k}_{\pi}\right)\right|}\right),
\end{gathered}
$$

and $h_{N}= \pm 1$.

For the process (14), the differential cross section reads

$$
d \sigma \sim \frac{1}{2}{\overline{\left|\mathcal{M}_{f i}\right|}}^{2}\left(1+\mathcal{P}_{l}^{\mu} \xi_{\mu}\right)
$$

while for the process (15) the cross section has the form

$$
d \sigma \sim \frac{1}{2}{\overline{\mid \mathcal{M}_{f i}}}^{2}\left(1+\mathcal{P}_{N}^{\mu} \zeta_{\mu}\right)
$$

where $\overline{\left|\mathcal{M}_{f i}\right|}$ is summed over the spins.

The four-vectors $\mathcal{P}_{\mu}^{l}$ and $\mathcal{P}_{\mu}^{N}$ describe the polarization of the charged lepton $(l)$ and the final nucleon $(N)$. The components of polarization are given by the ratio $[28,41]$

$$
\mathcal{P}_{X}^{Y}=\frac{d \sigma\left(s_{X}^{\mu}\right)-d \sigma\left(-s_{X}^{\mu}\right)}{d \sigma\left(s_{X}^{\mu}\right)+d \sigma\left(-s_{X}^{\mu}\right)}=\mathcal{P}_{Y}^{\mu} s_{X \mu},
$$


where $X=L$ (longitudinal), $N$ (normal), and $T$ (transverse) components of the polarization of the final fermion; $Y=l, N ;$ and $s=\xi, \zeta$.

Finally we define the degree of polarization of the particle:

$$
\mathcal{P}=\sqrt{\mathcal{P}_{L}^{2}+\mathcal{P}_{N}^{2}+\mathcal{P}_{T}^{2}}
$$

Notice that $0<\mathcal{P}<1$. If $\mathcal{P} \approx 0$, then the particle is unpolarized, while for a fully polarized particle, $\mathcal{P} \approx 1$.

\section{SPP MODELS}

We consider the interactions of the neutrinos of energy of the order of $1 \mathrm{GeV}$ with the free nucleon target. The dominant RES contribution is given by the weak $N \rightarrow \Delta(1232)$ transition. In both the HNV and FN models, the $N \rightarrow \Delta(1232)$ transition is described by the same formalism, but in the FN model, the weak vertex $N W^{+} \Delta$ is oversimplified. In phenomenological approaches, the NB contribution is described by some number of diagrams allowed by a symmetry; however, in the FN model the number of diagrams is smaller. In the next two subsections, the main features of both descriptions are shortly reviewed.

\section{A. HNV model}

We consider the HNV model as it is formulated in [8]; however, we notice the latest developments of the approach in Refs. [42,43]. The NB contribution is described by five diagrams; see Fig. 2. They are determined from the nonlinear sigma model. The main idea was to obtain, on the tree level, the axial and the vector currents from the nonlinear sigma model and to associate them with their phenomenological counterparts. The resonance contribution is given by two diagrams describing the weak $N \rightarrow \Delta(1232)$ transition. The $\Delta(1232)$ resonance is modeled by the 3/2-spin Rarita-Schwinger field [44].

\section{Nonresonant background}

The NB contribution is generated by five hadron currents:

$$
\begin{gathered}
r_{\mathrm{NP}}^{\mu}=-\frac{i g_{A}}{\sqrt{2} f_{\pi}} \not k_{\pi} \gamma_{5} S_{N}(p+q) \Gamma_{N}^{\mu}(q) \\
r_{\mathrm{CNP}}^{\mu}=-\frac{i g_{A}}{\sqrt{2} f_{\pi}} \Gamma_{N}^{\mu}(q) S_{N}\left(p^{\prime}-q\right) \not_{\pi} \gamma_{5} \\
r_{\mathrm{CT}}^{\mu}=-\frac{i}{\sqrt{2} f_{\pi}} \gamma^{\mu}\left(g_{A} F_{1}^{V}(q) \gamma_{5}-F_{\rho}\left(q-k_{\pi}\right)\right)
\end{gathered}
$$

$$
\begin{aligned}
& r_{\mathrm{PP}}^{\mu}=-\frac{i}{\sqrt{2} f_{\pi}} F_{\rho}\left(q-k_{\pi}\right) \frac{q^{\mu}}{q^{2}-m_{\pi}^{2}} \not \\
& r_{\mathrm{PF}}^{\mu}=-F_{1}^{V} \frac{2 i g_{A} M}{\sqrt{2} f_{\pi}} \frac{2 k_{\pi}^{\mu}-q^{\mu}}{\left(k_{\pi}-q\right)^{2}-m_{\pi}^{2}} \gamma_{5},
\end{aligned}
$$

where

$$
S_{N}(P)=\frac{\not P+M}{P^{2}-M^{2}}
$$

is the nucleon propagator.

The electroweak nucleon vertex reads

$$
\begin{aligned}
\Gamma_{N}^{\mu}(q)= & F_{1}^{V}(q) \gamma^{\mu}+i F_{2}^{V}(q) \frac{\sigma^{\mu \nu} q_{\nu}}{2 M} \\
& -G_{A}(q)\left(\gamma^{\mu}+\frac{\not q q^{\nu}}{m_{\pi}^{2}-q^{2}}\right) \gamma_{5},
\end{aligned}
$$

where $F_{k}^{V}\left(q^{2}\right)$ is the vector nucleon form factor [see (B1)], $G_{A}$ is the axial nucleon form factor [see (B2)], $g_{A}=1.26$ is the axial nucleon coupling, and the form factor $F_{\rho}$ is given by (B7).

\section{2. $\Delta(1232)$ contribution}

The $N \rightarrow \Delta(1232)$ resonance transition currents have the form

$$
\begin{gathered}
r_{\Delta P}^{\mu}=i \sqrt{3} \frac{f^{*}}{m_{\pi}} k_{\pi}^{\alpha} S_{\alpha \beta}(p+q) \Gamma^{\beta \mu}(p, q) \\
r_{C \Delta P}^{\mu}=\frac{i}{\sqrt{3}} \frac{f^{*}}{m_{\pi}} \gamma^{0}\left[\Gamma^{\alpha \mu}\left(p^{\prime},-q\right)\right]^{\dagger} \gamma^{0} k_{\pi}^{\beta} S_{\alpha \beta}\left(p^{\prime}-q\right),
\end{gathered}
$$

where $f^{*}=2.14$.

$$
\begin{aligned}
S_{\alpha \beta}(p)= & -\frac{\left(\not p+M_{\Delta}\right)}{p^{2}-M_{\Delta}^{2}+i M_{\Delta} \Gamma_{\Delta}(p)} \\
& \times\left(g_{\alpha \beta}-\frac{1}{3} \gamma_{\alpha} \gamma_{\beta}-\frac{2}{3} \frac{p_{\alpha} p_{\beta}}{M_{\Delta}^{2}}+\frac{1}{3} \frac{p_{\alpha} \gamma_{\beta}-\gamma_{\alpha} p_{\beta}}{M_{\Delta}}\right)
\end{aligned}
$$

is the 3/2-spin particle propagator, $M_{\Delta}=1232 \mathrm{MeV}$, while $\Gamma_{\Delta}(p)$ is the resonance width, which in the HNV model is given by (C1).

The electroweak vertex for the $W^{+}(q) N(p) \rightarrow \Delta(p+q)$ transition has the vector + axial form, namely,

$$
\Gamma^{\nu \mu}(p, q)=\left[V_{3 / 2}^{\nu \mu}(p, q)+A_{3 / 2}^{\nu \mu}(p, q)\right] \gamma_{5} .
$$

The vector part of (41) reads [45] 

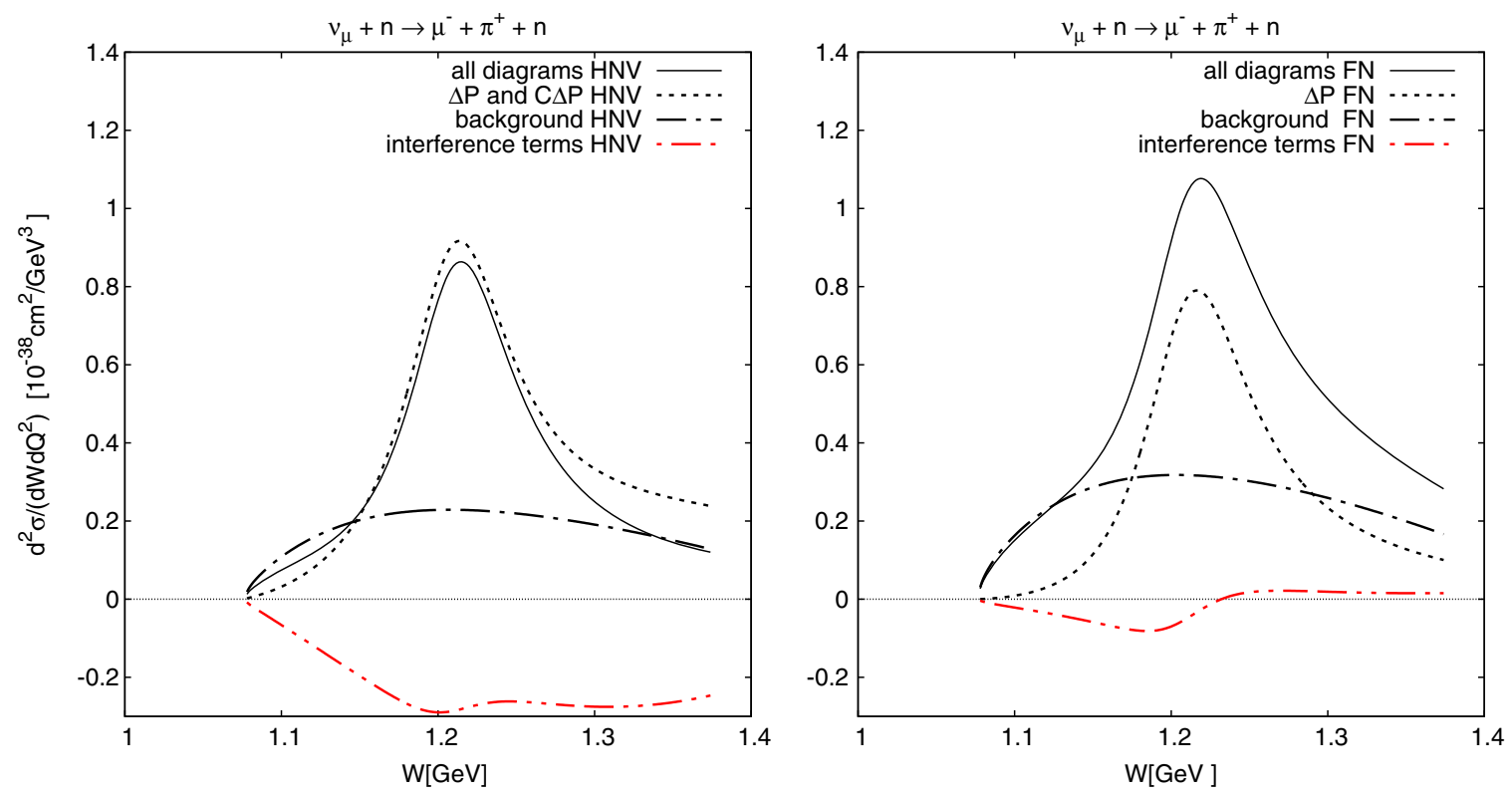

FIG. 3. The differential cross section and its pure resonance (dotted line), pure nonresonant (dashed-dotted), and the interference between resonant and nonresonant amplitude (dashed-dotted-dotted) contributions in the HNV (left) and FN (right) models. The pure resonance contribution is given by $\left|\mathcal{M}_{\Delta P}+\mathcal{M}_{C \Delta P}\right|^{2}$ and $\left|\mathcal{M}_{\Delta P}\right|^{2}$ in the HNV and FN, models respectively. The neutrino energy $E=0.7 \mathrm{GeV}$ and $Q^{2}=0.1 \mathrm{GeV}^{2}$.

$$
\begin{aligned}
V_{3 / 2}^{\nu \mu}(p, q)= & \frac{C_{3}^{V}}{M}\left(g^{\nu \mu} \phi 1-q^{\nu} \gamma^{\mu}\right) \\
& +\frac{C_{4}^{V}}{M^{2}}\left(g^{\nu \mu} q \cdot(p+q)-q^{\nu}\left(p^{\mu}+q^{\mu}\right)\right) \\
& +\frac{C_{5}^{V}}{M^{2}}\left(g^{\nu \mu} q \cdot p-q^{\nu} p^{\prime \mu}\right) .
\end{aligned}
$$

The vector form factors are given by the fit form [46]; see Appendix B 1.

The axial part of the vertex (41) reads [47]

$$
\begin{aligned}
A_{3 / 2}^{\nu \mu}(p, q)= & \left(\frac{C_{3}^{A}}{M}\left(g^{\nu \mu} \phi-q^{\nu} \gamma^{\mu}\right)\right. \\
& +\frac{C_{4}^{A}}{M^{2}}\left(g^{\nu \mu} q \cdot(p+q)-q^{\nu}\left(p^{\mu}+q^{\mu}\right)\right) \\
& \left.+g^{\nu \mu} C_{5}^{A}+\frac{C_{6}^{A}}{M^{2}} q^{\nu} q^{\mu}\right) \gamma_{5} .
\end{aligned}
$$

The axial form factors are obtained from the analysis of the neutrino scattering data. However, the data are not accurate enough to get information about the four independent form factors [48]. Therefore the following simplifications are made:

(i) $C_{3}^{A}\left(Q^{2}\right)=0$, as it is suggested by simple quark model (see, e.g., [49]); (ii)

$$
C_{4}^{A}\left(Q^{2}\right)=-\frac{C_{5}^{A}\left(Q^{2}\right)}{4}
$$

as it is supported by dispersion analyses [9];

(iii) $C_{6}^{A}$ is a function of $C_{5}^{A}$, namely,

$$
C_{6}^{A}\left(Q^{2}\right)=\frac{M^{2}}{m_{\pi}^{2}+Q^{2}} C_{5}^{A}\left(Q^{2}\right),
$$

as it is obtained from the partially conserved axial current (PCAC) hypothesis.

For the numerical analyses, we take the $C_{5}^{A}$ fit from [46]; see Appendix B 1.

\section{B. Fogli-Nardulli model}

The FN model ${ }^{1}$ formulated in [11] describes the pion production in the first and second resonance regions. But in this paper we consider only the first resonance region.

\section{Nonresonant background}

The NB contribution is described by three diagrams: the pion in flight (PF), the same as in the HNV model, and two nucleon pole diagrams, NP and CNP. But in the latter,

\footnotetext{
${ }^{1} \mathrm{~A}$ short review of the $\mathrm{FN}$ model can be found in [50].
} 
the pseudoscalar pion-nucleon coupling was implemented, while in the HNV model the pseudovector pion-nucleon coupling is discussed:

$$
\begin{gathered}
\tilde{r}_{\mathrm{NP}}^{\mu}=i \sqrt{2} g_{N N \pi} \gamma_{5} S_{N}(p+q) \Gamma_{N}^{\mu}(q) \\
\tilde{r}_{\mathrm{CNP}}^{\mu}=i \sqrt{2} g_{N N \pi} \Gamma_{N}^{\mu}(q) S_{N}\left(p^{\prime}-q\right) \gamma_{5} \\
\tilde{r}_{\mathrm{PF}}^{\mu}=-i \sqrt{2} g_{N N \pi} \gamma_{5} \frac{2 k_{\pi}^{\mu}-q^{\mu}}{\left(k_{\pi}-q\right)^{2}-m_{\pi}^{2}} F_{\pi}\left(k_{\pi}-q\right),
\end{gathered}
$$

where $g_{N N \pi}=\sqrt{14.8 \cdot 4 \pi}$ (see, e.g., [51]), and the $F_{\pi}$ form factor is given in Appendix B 1.
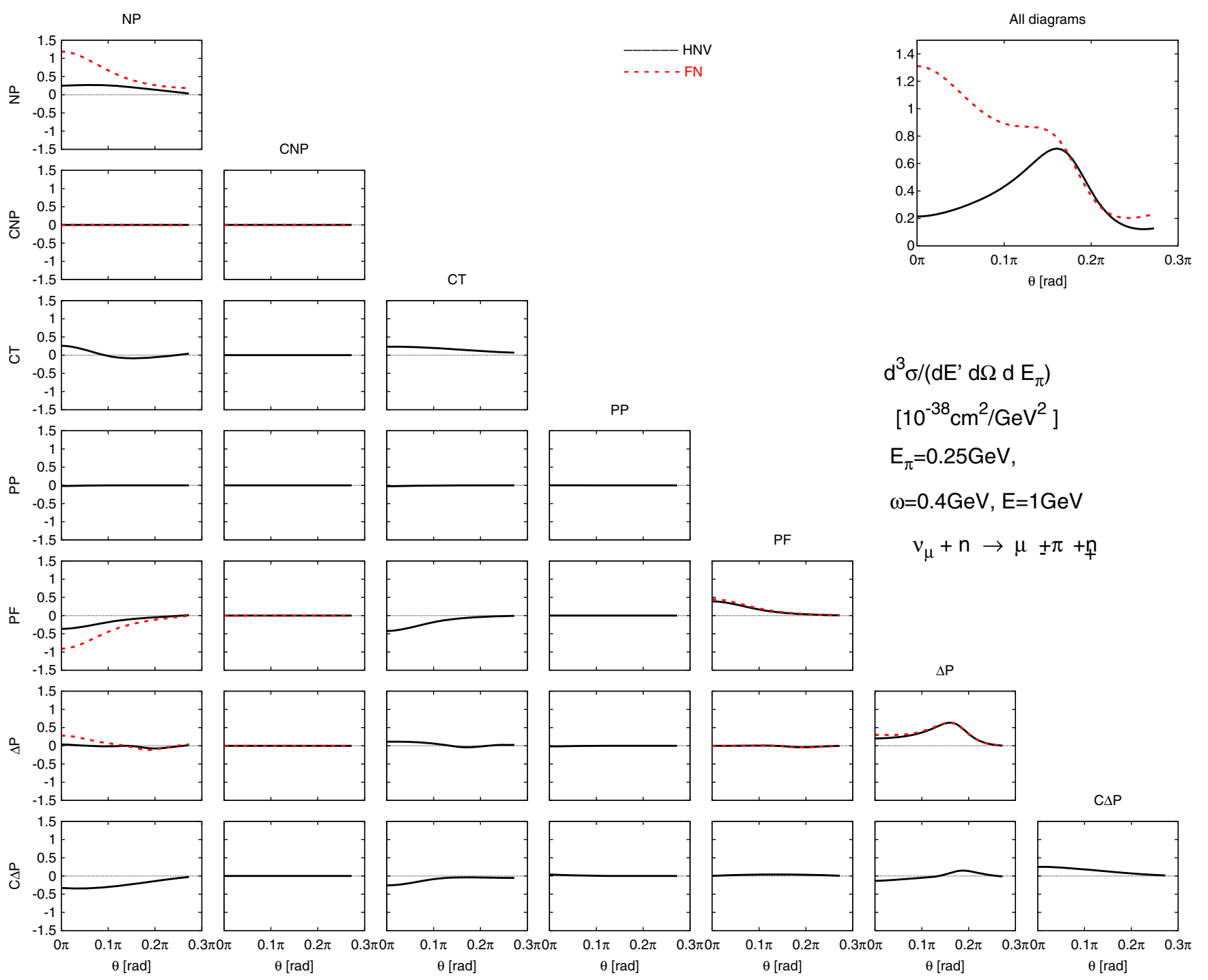

FIG. 4. Separation of the differential cross section into various interference components. The solid black (dotted red) line represents the HNV (FN) model predictions. The contribution from the $\left|\mathcal{M}_{a}\right|^{2}$ is on the diagonal, while below the diagonal the interference terms $2 \Re\left(\mathcal{M}_{i} \mathcal{M}_{j}^{*}\right)$ are plotted ( $j$ indicates the column and $i$ the row). 

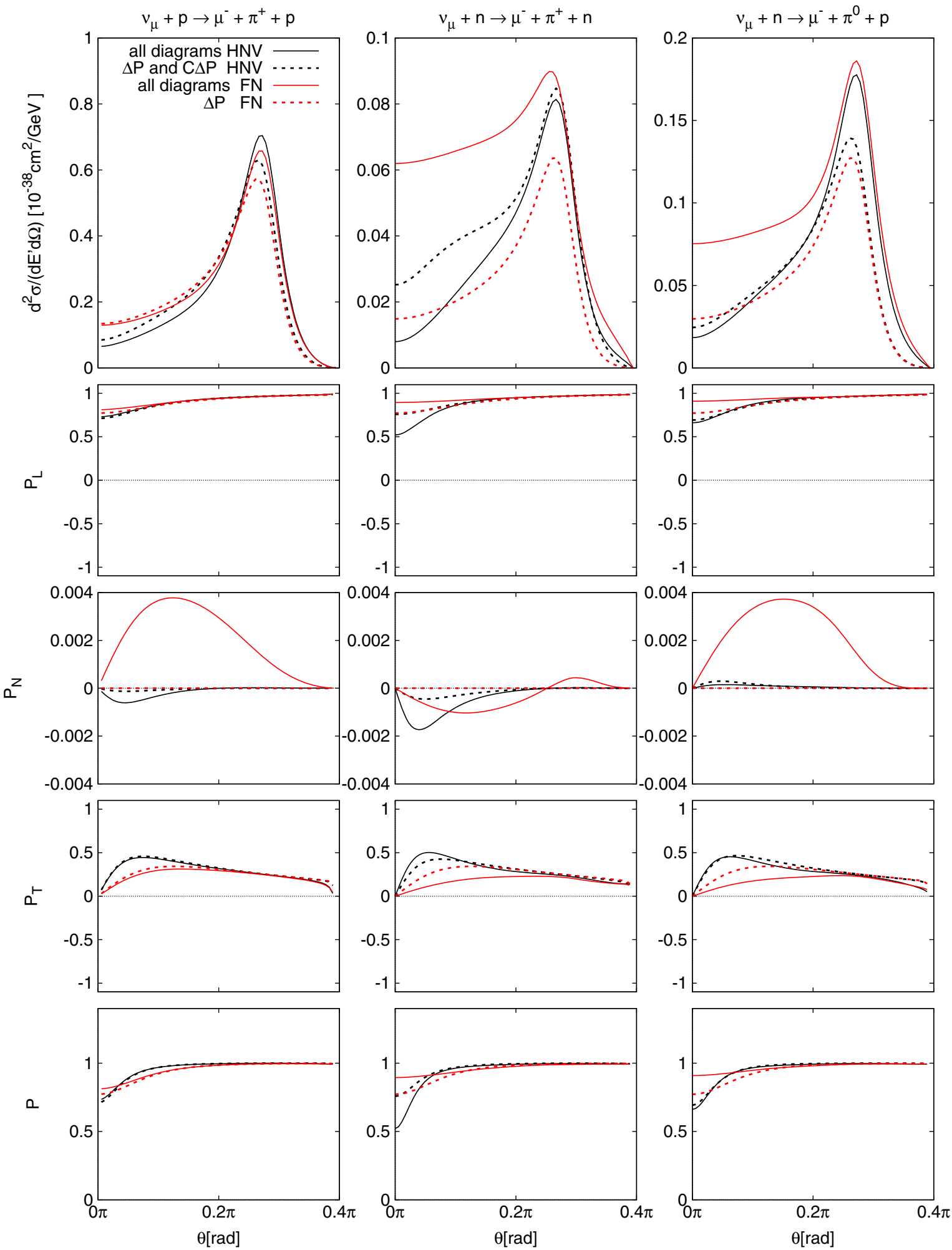

FIG. 5. Angular dependence of the components of $\mathcal{P}_{\mu^{-}}^{\alpha}(E, \omega, \theta)$ vector polarization and the degree of polarization (last row) of the $\mu^{-}$ lepton, for the energy of the neutrino $E=1 \mathrm{GeV}$ and the transfer of energy $\omega=0.5 \mathrm{GeV}$. The dotted (solid) line represents the RES (full model) contributions of the HNV (black) and FN (red) models. The resonance contribution is given by $\left|\mathcal{M}_{\Delta P}+\mathcal{M}_{C \Delta P}\right|^{2}$ and $\left|\mathcal{M}_{\Delta P}\right|^{2}$ in the HNV and FN models, respectively. 

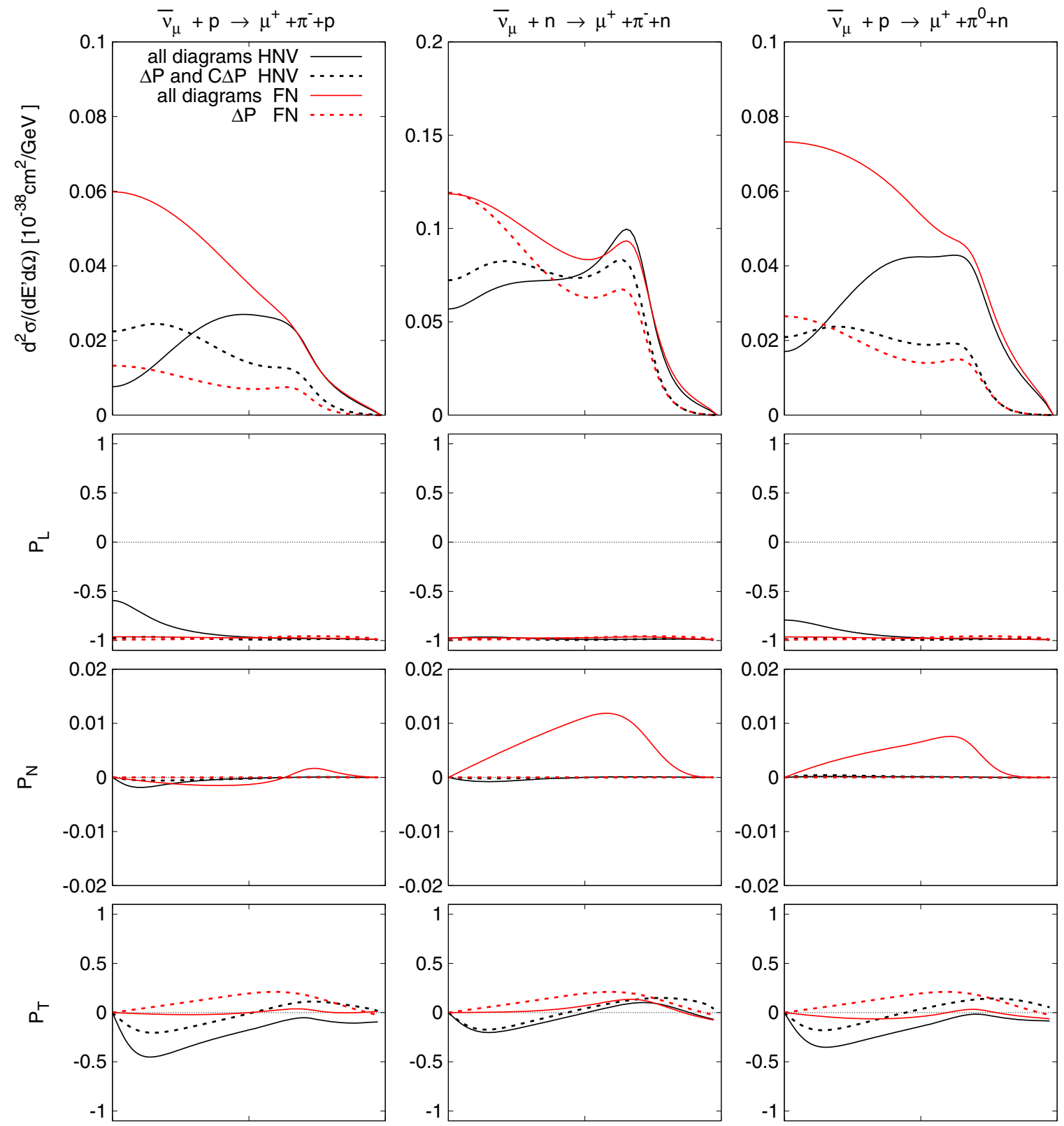

口.
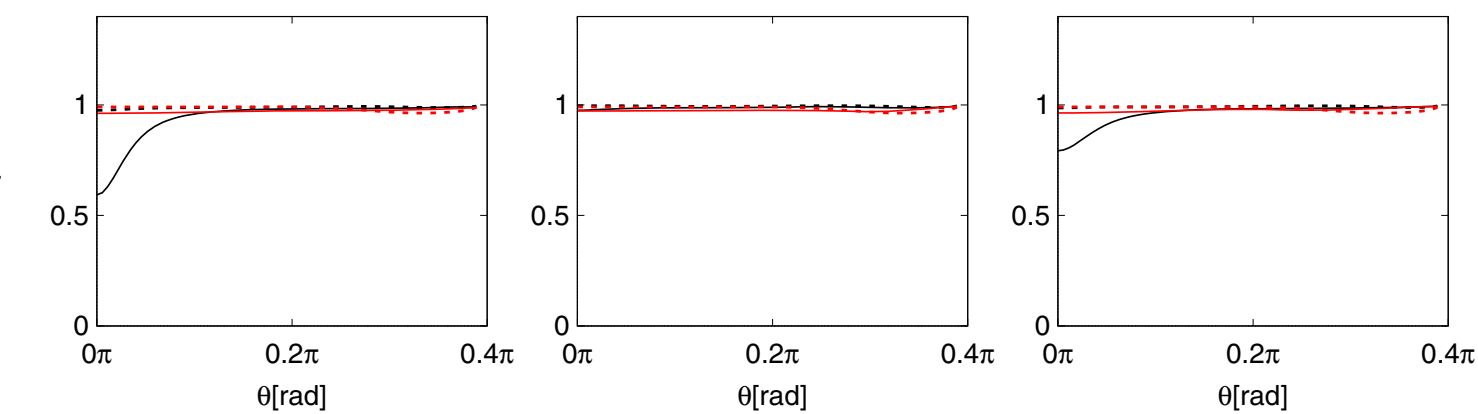

FIG. 6. Angular dependence of the components of $\mathcal{P}_{\mu^{+}}^{\alpha}(E, \omega, \theta)$ vector polarization and the degree of polarization (last row) of the $\mu^{+}$ lepton, for the energy of the neutrino $E=1 \mathrm{GeV}$ and the transfer of energy $\omega=0.5 \mathrm{GeV}$. The dotted (solid) line represents the RES (full model) contributions of the HNV (black) and FN (red) models. The resonance contribution is given by $\left|\mathcal{M}_{\Delta P}+\mathcal{M}_{C \Delta P}\right|^{2}$ and $\left|\mathcal{M}_{\Delta P}\right|^{2}$ in the HNV and FN models, respectively. 

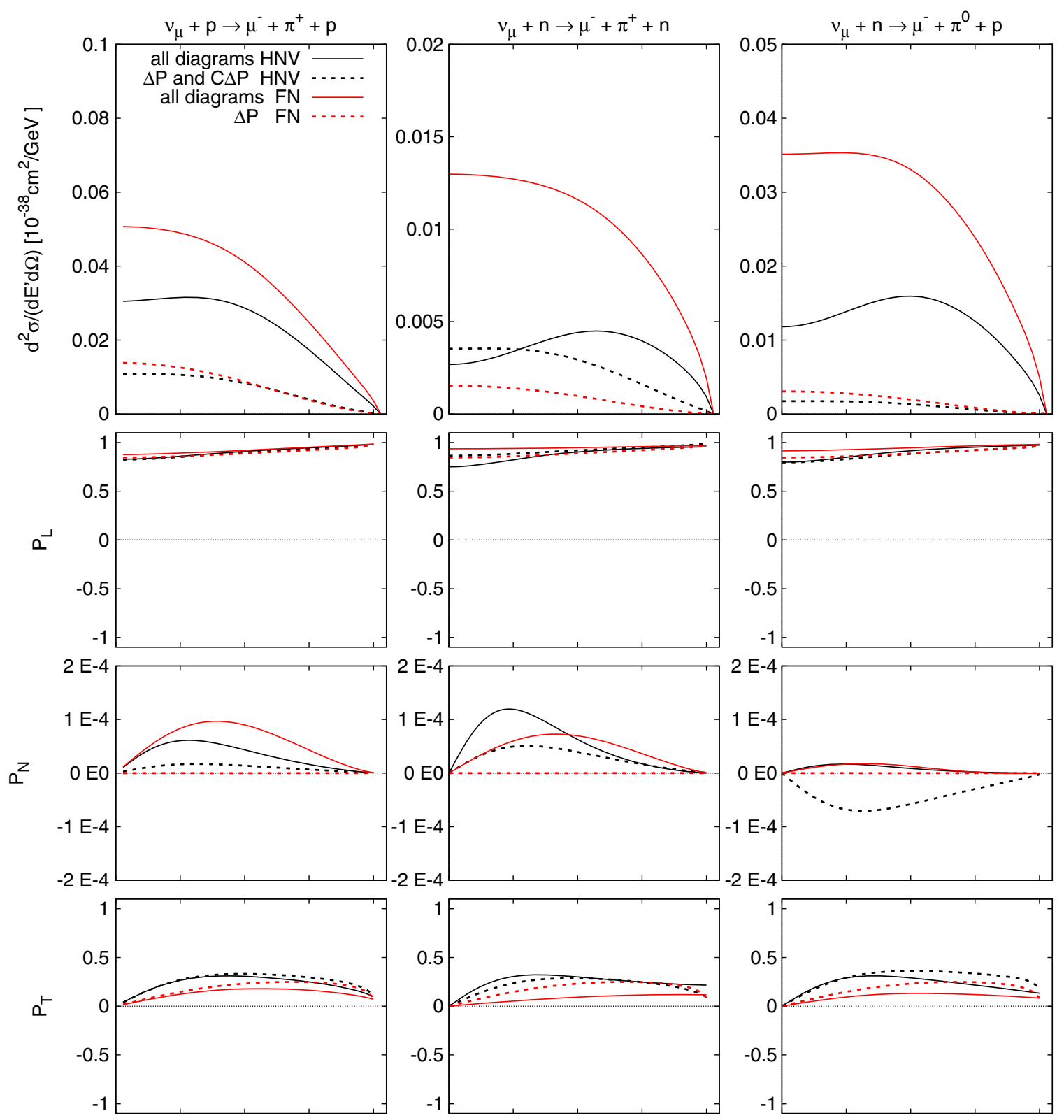

口
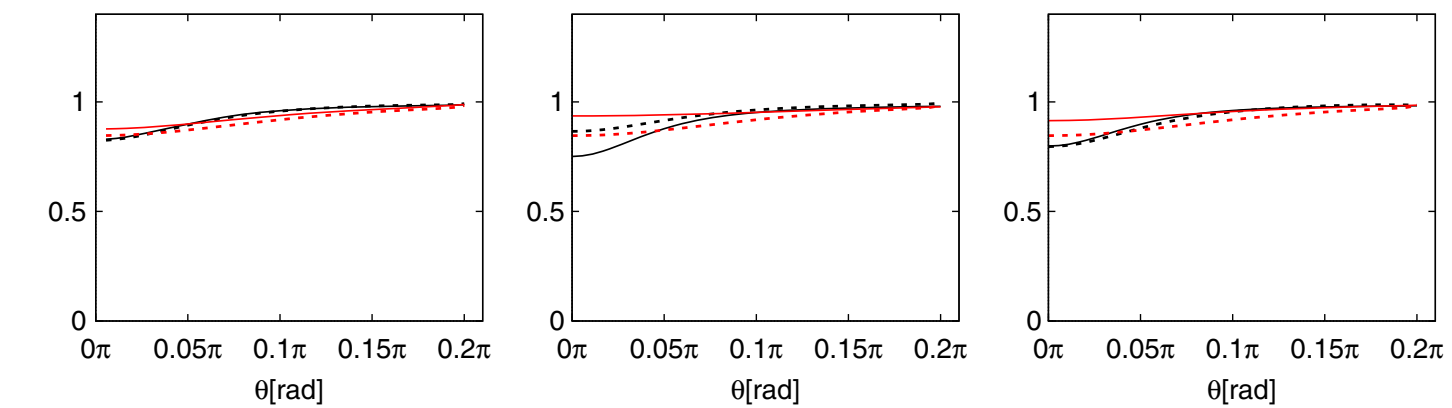

FIG. 7. The same as Fig. 5, but for $E=0.6 \mathrm{GeV}$. 

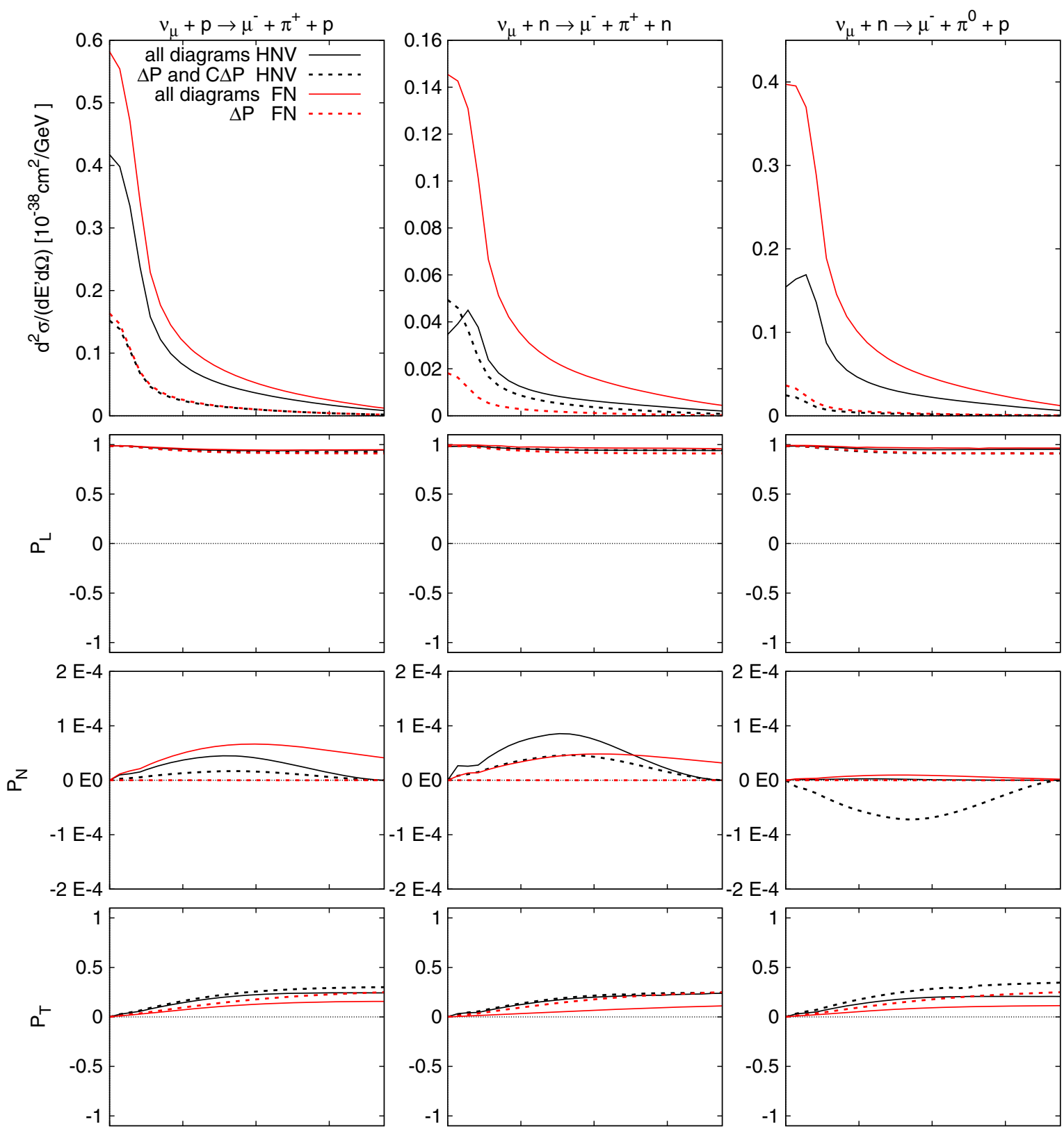

a.
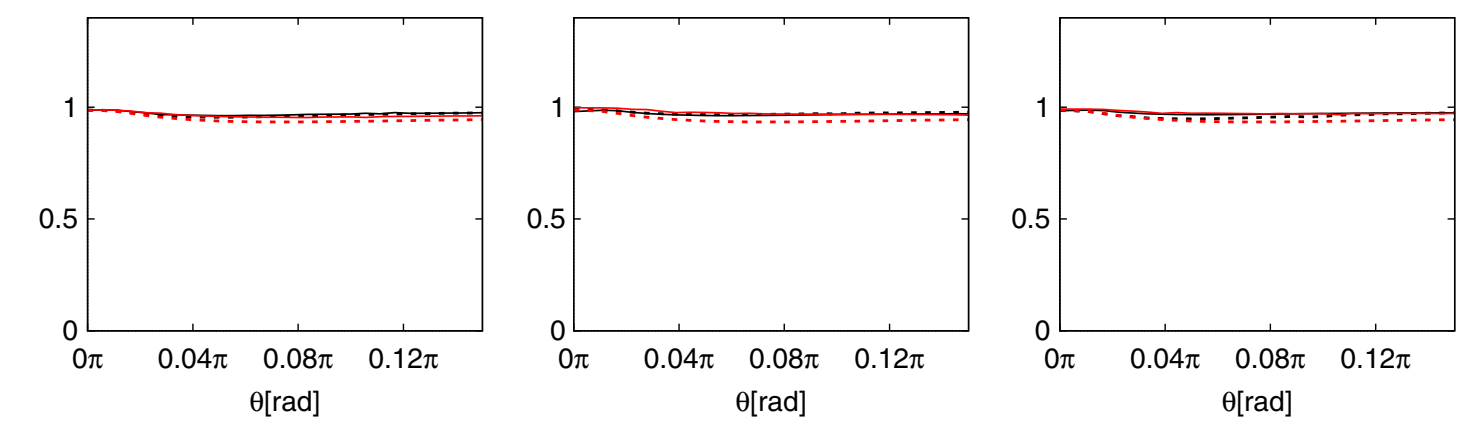

FIG. 8. The same as Fig. 5, but the predictions are T2K-flux averaged. 


$$
C_{4}^{V}\left(Q^{2}\right)=-\frac{M}{W} C_{3}^{V}
$$

The width of the $\Delta(1232)$ resonance is given by (C3).

\section{NUMERICAL RESULTS}

\section{A. Unpolarized cross sections}

Most of the SPP models, including the HNV and FN approaches, reproduce the cross section data for the $\nu_{\mu} p \rightarrow \mu^{-} p \pi^{+}$process with reasonable accuracy. The agreement with the data is achieved by appropriately tuning the values of the parameters of the $C_{5}^{A}$ axial form factor $[11,16,46,50,52]$. The main problem is to obtain coherent model predictions for all three CC channels. Indeed, the ANL data for the $\nu n \rightarrow \mu^{-} n \pi^{+}$process seem to be inconsistent with the other channels [46]. This can be caused by oversimplified treatment of the deuteron structure effects in the analysis of the ANL data (see [53]), incomplete description of the $\Delta(1232)$ resonance propagation (see [43]), and/or low quality of the data.

It seems that critical studies of the SPP models can be performed only if new, more precise measurements of the interactions of the neutrinos with the free nucleon target will be delivered. Moreover, the information about the SPP dynamics hidden in the total and single differential cross sections (like $d \sigma / d Q^{2}$ ) and even double differential cross sections is limited because important features of an approach are integrated out. Hence, the analysis of these cross-section data does not allow us to verify which model, among many in the market, is the closest in description to reality. It is illustrated in Fig. 3, where we plot the $d^{2} \sigma / d W d Q^{2}$ distribution and its partition into

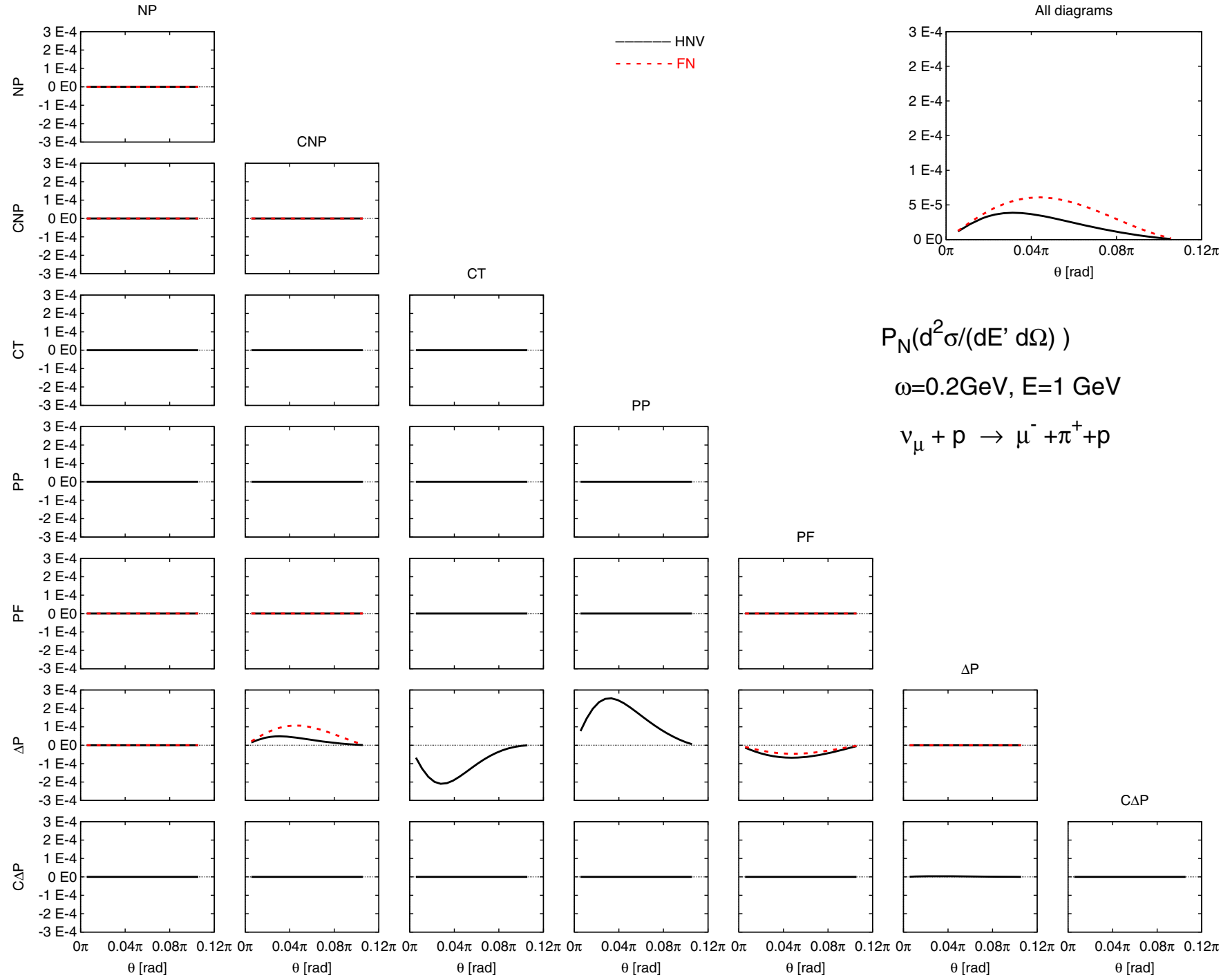

FIG. 9. Separation of the normal component of the polarization of $\mu^{-}$into various interference contributions. The solid black (dotted red) line represents the HNV (FN) model predictions. The contribution from the $\left|\mathcal{M}_{a}\right|^{2}$ are on the diagonal while below the diagonal the interference terms $2 \Re\left(\mathcal{M}_{i} \mathcal{M}_{j}^{*}\right)$ are plotted ( $j$ indicates the column and $i$ the row). 
(i) pure resonance contribution;

(ii) interference between the RES and the NB amplitudes;

(iii) pure NB contribution.

Although the RES and NB contribute differently in both models, the shape and the magnitude of $d^{2} \sigma / d W d Q^{2}$ obtained within both approaches are very similar. The significant disparities between the predictions of both models manifest when the triple differential cross sections are examined. In Fig. 4 we plot $d^{3} \sigma / d E^{\prime} d \Omega d E_{\pi}$ and its partition into all possible interference terms calculated at particular kinematics. At a low scattering angle, the FN cross section increases rapidly while the HNV model predictions fall down. The low- $\theta$ behavior of the FN's cross section is determined by a $\left|\mathcal{M}_{\mathrm{NP}}\right|^{2}$ contribution. In the HNV model, the contribution from the NP diagram is smaller and it is suppressed by the contribution missing in the FN model, namely, the interference terms: $\mathcal{M}_{C \Delta P} \mathcal{M}_{\mathrm{NP}}^{*}$, $\mathcal{M}_{\mathrm{CT}} \mathcal{M}_{\mathrm{PF}}^{*}$, and others.

Certainly inspection of the triple differential cross sections, and their dependence on the $\phi_{\pi}$ angle, may deliver valuable information about the RES and NB dynamics. Let us also mention that additional constraints on the NB contribution can be obtained from the combined analysis of various mass distributions. It is demonstrated in Ref. [16], where a detailed discussion of the $W(N \pi), W(\mu N)$, and $W(\mu \pi)$ event distributions of the ANL and BNL experiments is given. However, in the next section we shall demonstrate that the PT observables contain unique information about the dynamics of the SPP.

\section{B. Polarization transfer observables}

We start the presentation of the PT results from the discussion of the polarization properties of the charged lepton produced in process (14). In Figs. 5 and 6, the longitudinal, normal, and transverse components, as well as the degree of polarization of the $\mu^{-}$and $\mu^{+}$leptons, are shown. Additionally, Fig. 7 includes the plots of $\mu^{-}$ polarization components calculated for an averaged $\mathrm{T} 2 \mathrm{~K}$ [3] experiment neutrino energy, $E=0.6$. In Fig. 8, the polarization components of the $\mu^{-}$lepton averaged over the T2K energy flux [54] are shown. The muon lepton is a light particle and hence it should be almost polarized. However, we notice that for some kinematics, namely, at a low scattering angle, the $\mu^{ \pm}$lepton can be partially polarized.

Unique information about the SPP dynamics is hidden in the normal component of the polarization. Indeed, this observable is dominated by the interference of the $\mathcal{M}_{\Delta P}$ and $\mathcal{M}_{C \Delta P}$ amplitudes with the NB diagrams. Therefore, the sign of the normal component is defined by the relative sign between the RES and NB contributions. This property is illustrated in Fig. 9, where we plot the partition of the normal component into all interference terms.
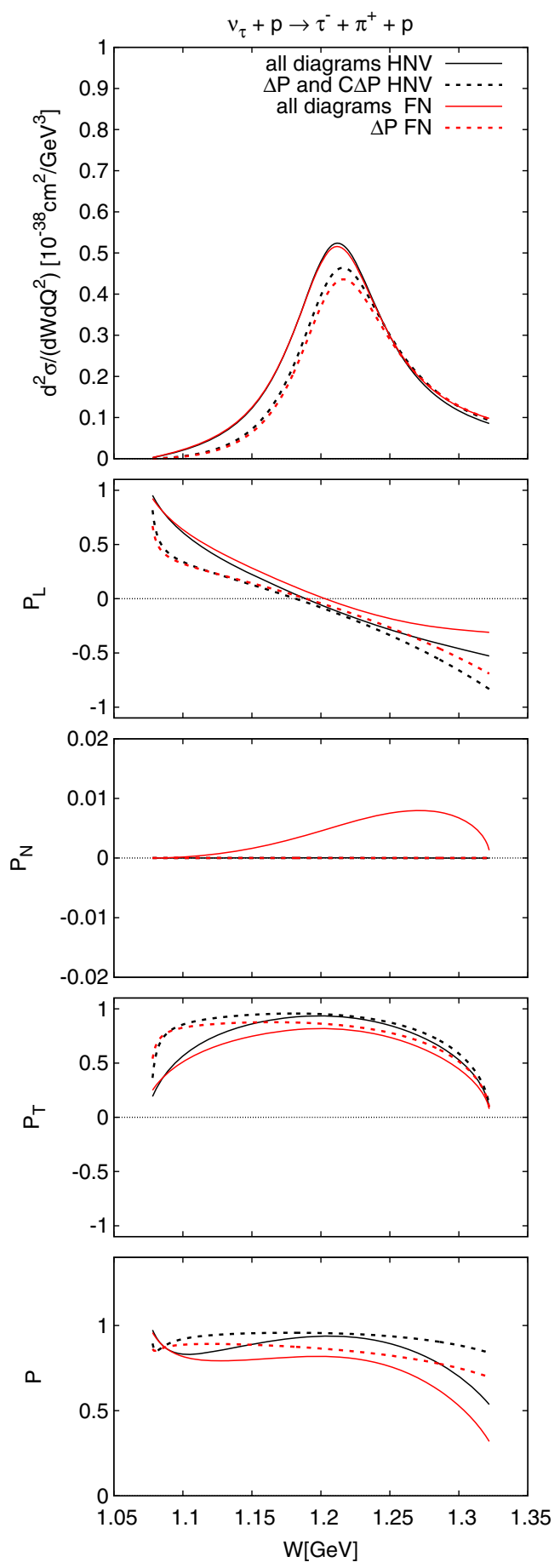

FIG. 10. $W$ dependence of the components of the $\mathcal{P}_{\tau^{-}}^{\alpha}\left(E, Q^{2}, W\right)$ vector polarization and the degree of polarization (last row) of the $\tau^{-}$lepton, for the energy of the neutrino $E=$ $5 \mathrm{GeV}$ and $Q^{2}=1 \mathrm{GeV}^{2}$. The dotted (solid) line represents the RES (full model) contributions of the HNV (black) and FN (red) models. The resonance contribution is given by $\left|\mathcal{M}_{\Delta P}+\mathcal{M}_{C \Delta P}\right|^{2}$ and $\left|\mathcal{M}_{\Delta P}\right|^{2}$ in the HNV and FN models, respectively.

Now let us review the polarization properties the $\tau$ lepton produced in the $\nu_{\tau} N$ scattering. The mass of the $\tau$ is large; hence, it can be partially polarized. Indeed, in 

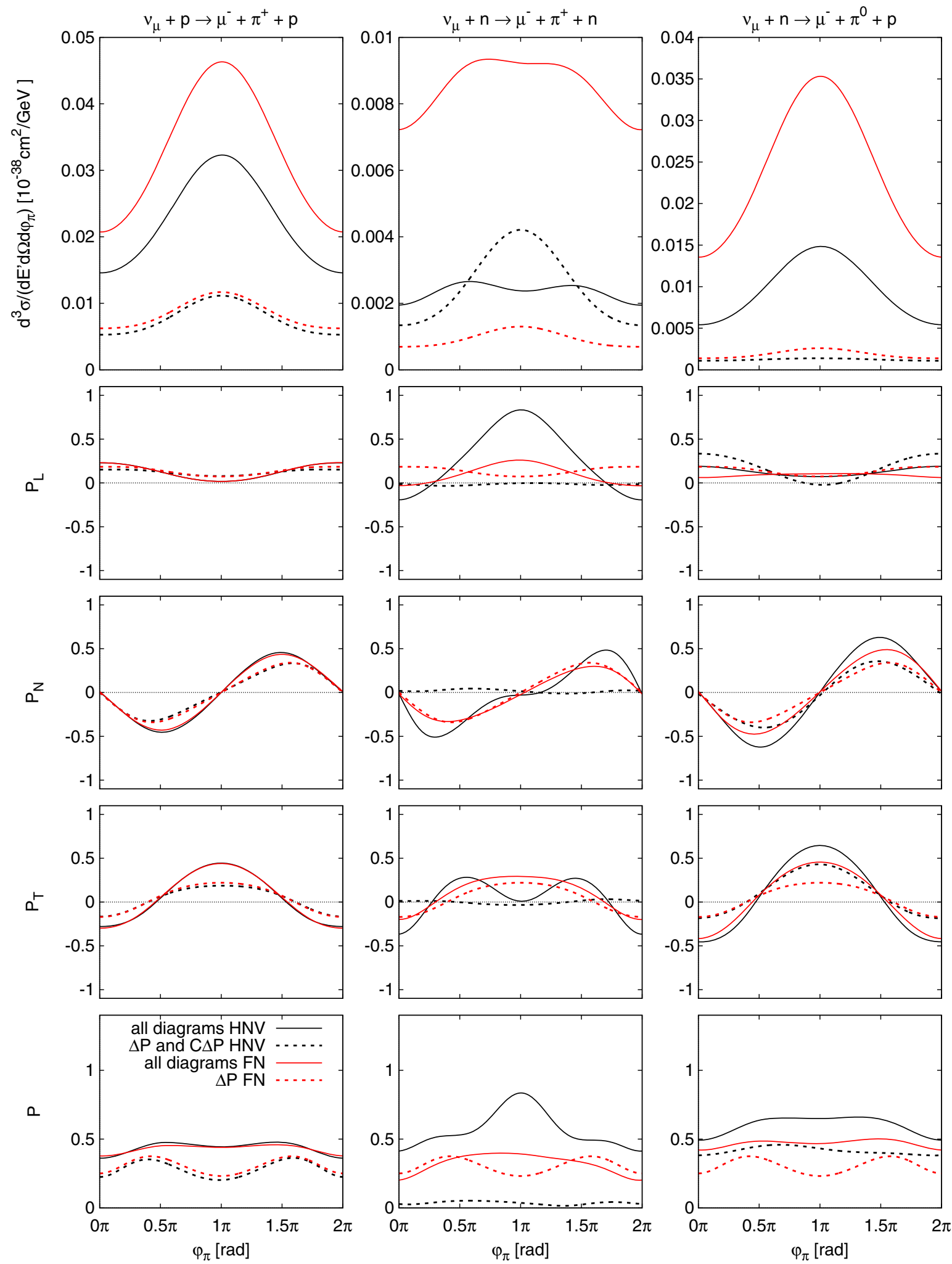

FIG. 11. Angular dependence of the components of $\mathcal{P}_{N}^{\alpha}\left(E, \theta, \omega, \phi_{\pi}\right)$ vector polarization and the degree of polarization (last row) of the nucleon, for the energy of the neutrino $E=1 \mathrm{GeV}$, the scattering angle $\theta=5^{\circ}$, and the energy transfer $\omega=0.2 \mathrm{GeV}$. The dotted (solid) line represents the RES (total) contributions of the HNV (black) and FN (red) models. The resonance contribution is given by $\left|\mathcal{M}_{\Delta P}+\mathcal{M}_{C \Delta P}\right|^{2}$ and $\left|\mathcal{M}_{\Delta P}\right|^{2}$ in the HNV and FN models, respectively. 
Fig. 10 we plot the predictions of the degree of polarization. It varies from 0.3 to 1.0 . Let us remark that the polarization vector of the $\tau$ lepton is one of the variables which describes the angular distribution of the products of its decay. The model dependence of the predictions of the polarization vector of the tau lepton on the SPP model assumptions seems to be stronger than in the case of the muon lepton.
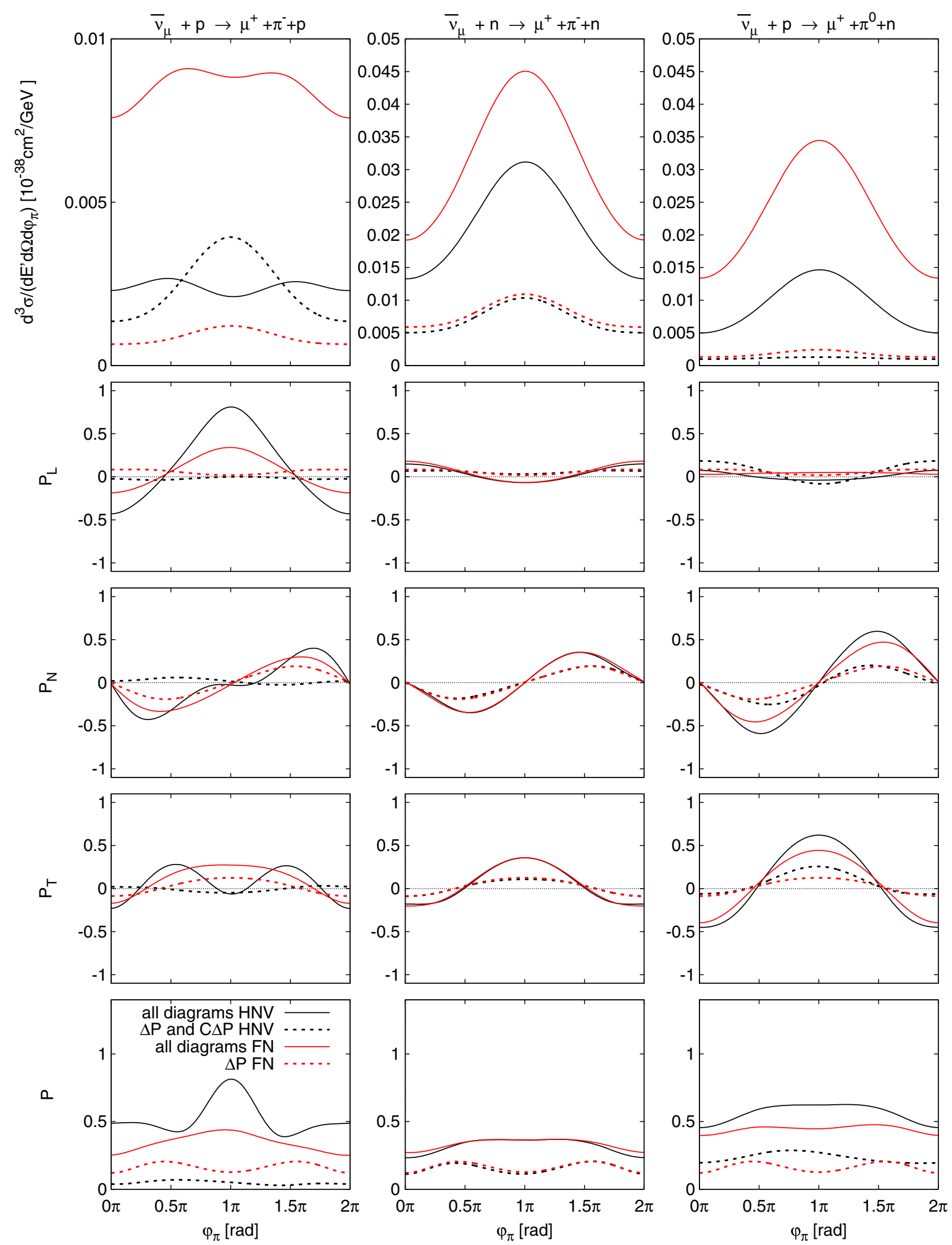

FIG. 12. The same as Fig. 11 , but for $\bar{\nu}_{\mu} N$ scattering. 
Interesting conclusions are obtained from the analysis of the polarization properties of the final nucleon produced in process (15). In Figs. 11 and 12, the angular dependence (in the $\phi_{\pi}$ angle) of the polarization components and the degree of polarization of the nucleon produced in the $\nu_{\mu} N$ and $\bar{\nu}_{\mu} N$ interactions are plotted. Similarly as for the discussion of the lepton polarization properties, we show also the predictions of the polarization components for the $\mathrm{T} 2 \mathrm{~K}$
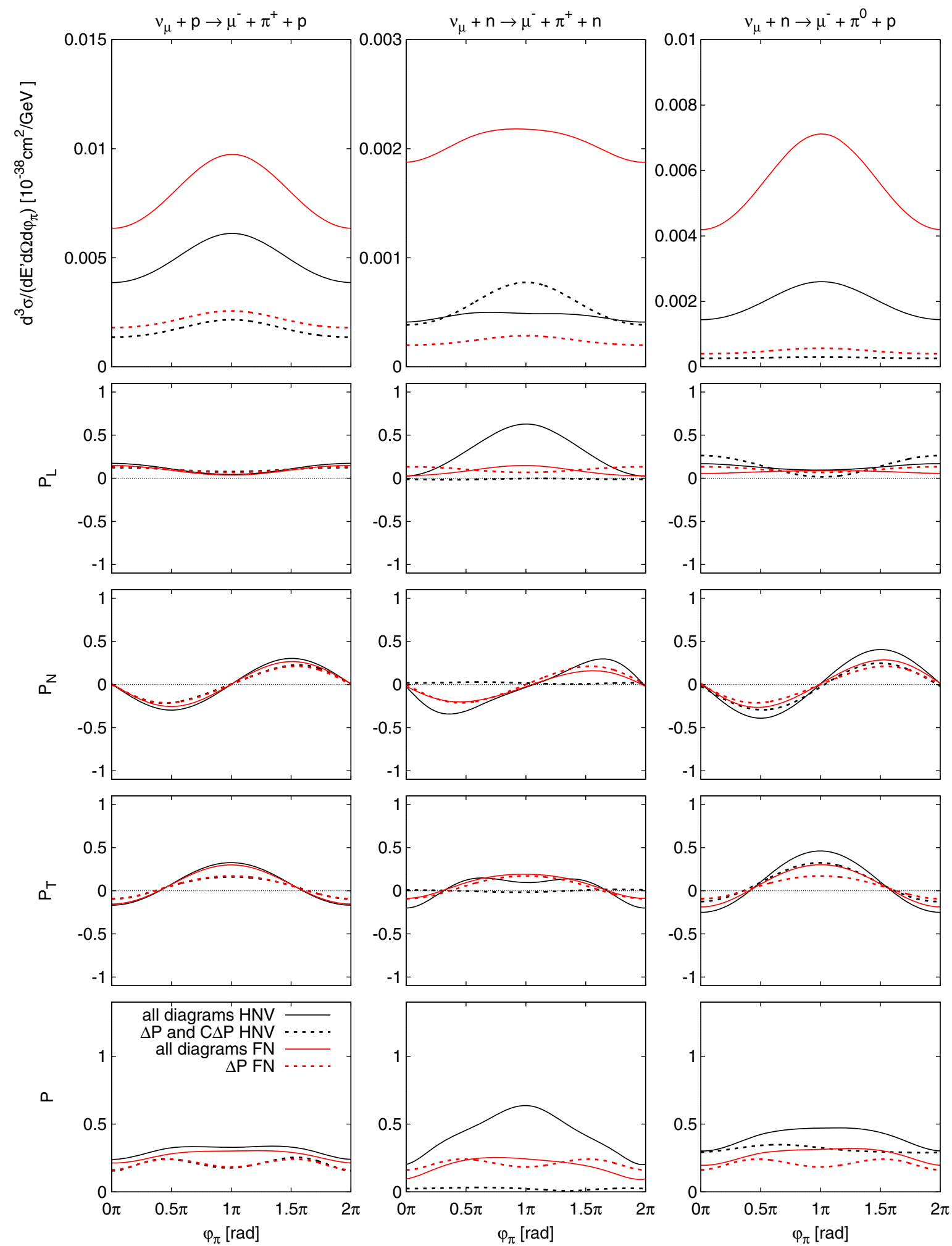

FIG. 13. The same as Fig. 11, but for the neutrino energy $E=0.6 \mathrm{GeV}$ and the energy transfer $\omega=0.2 \mathrm{GeV}$. 
neutrino averaged energy in Fig. 13 and for the T2K-flux averaged in Fig. 14. Notice that the resonance parts of the normal, transverse, and longitudinal polarization components have a sinusoidal character, which is distorted by the inclusion of the NB contribution. At some kinematics the normal polarization of the nucleon is large-it reaches the value 0.5 . Similarly as in the lepton polarization case, the interference of the RES with the NB diagrams gives a
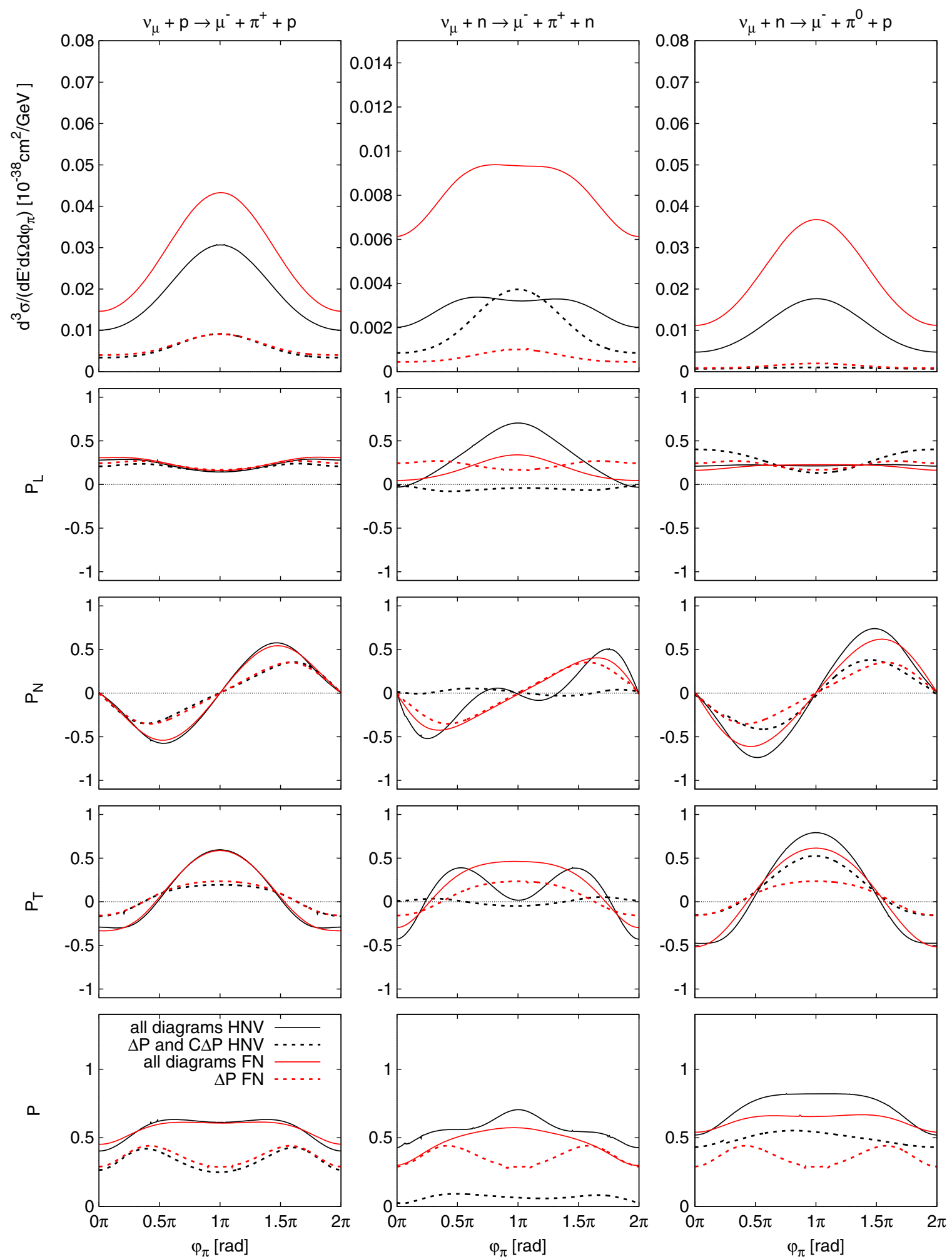

FIG. 14. The same caption as in Fig. 11 but T2K-flux averaged. 

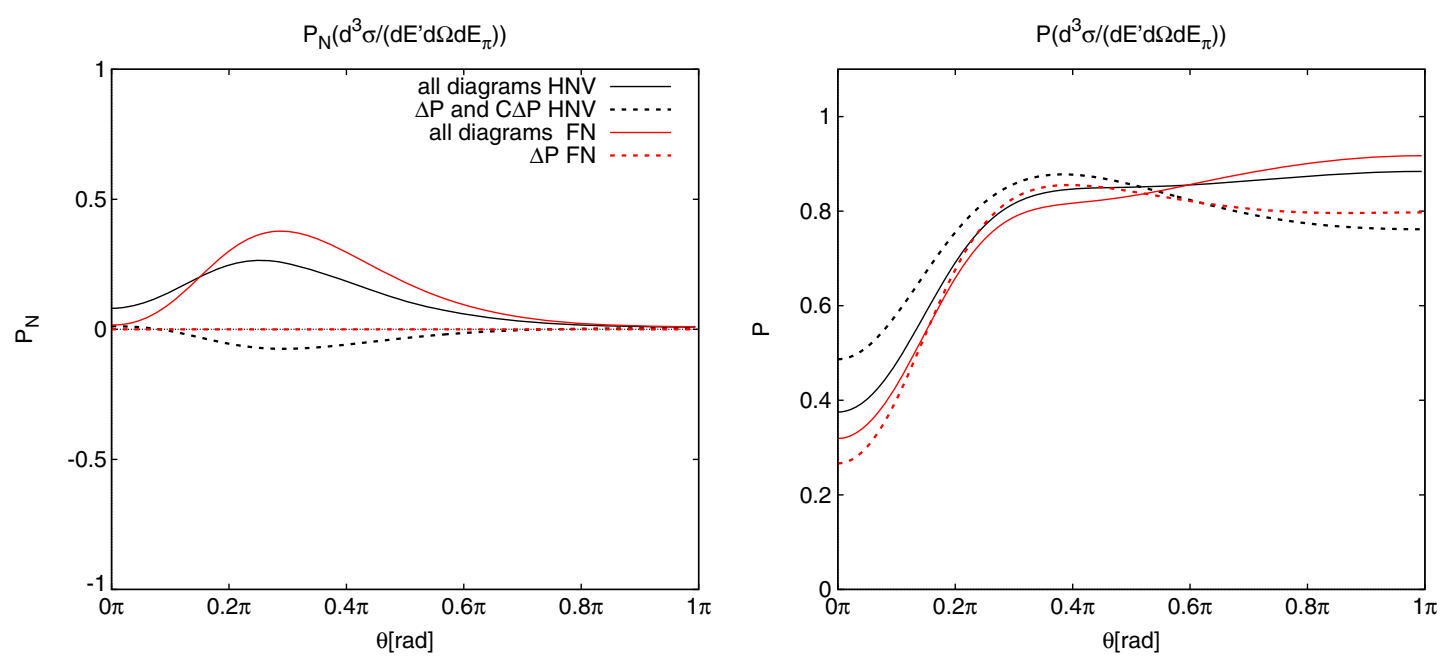

FIG. 15. The angular dependence in $\theta$ of the normal component of the polarization (left) and the degree of polarization (right) of the recoiled proton in the process $\nu_{\mu}+p \rightarrow \mu^{-}+p+\pi^{+}$. The predictions are obtained within the HNV (black) and FN (red) models. The RES (full model) contribution is denoted by the dotted (solid) line. The neutrino energy $E=0.7 \mathrm{GeV}$, the energy transfer $\omega=0.5 \mathrm{GeV}$, and the pion energy $E_{\pi}=0.25 \mathrm{GeV}$. The resonance contribution is given by $\left|\mathcal{M}_{\Delta P}+\mathcal{M}_{C \Delta P}\right|^{2}$ and $\left|\mathcal{M}_{\Delta P}\right|^{2}$ in the HNV and FN models, respectively.

sizable contribution, which for some kinematics becomes dominant. It is illustrated in Fig. 15.

\section{SUMMARY}

We discussed polarization properties of fermions produced in the SPP processes induced by the charged current neutrino-nucleon scattering. The components of the polarization vector of the outgoing charged lepton and the nucleon were calculated. In order to make the discussion more realistic, we have made the predictions of the polarization properties for the $\mathrm{T} 2 \mathrm{~K}$ experiment. It turned out that the PT observables are very sensitive on the details of the SPP model. In order to investigate how a change in a model's assumptions affects the predictions of the polarization components, we considered two SPP approaches. It was demonstrated that the most interesting information about the SPP dynamics is hidden in the normal component of the polarization of the outgoing lepton and the nucleon. In particular, the sign of the normal polarization of the charged lepton is determined by the relative sign between the NB and RES amplitudes.

Eventually we conclude that the investigation of the polarization transfer observables in the SPP in $\nu N$ scattering should deliver a complementary (to the spin-averaged cross sections) knowledge about the resonance and nonresonance contributions.

\section{ACKNOWLEDGMENTS}

All algebraic calculations have been performed using FORM symbolic language [55]. The calculations have been carried out at the Wroclaw Centre for Networking and Supercomputing (http://www.wcss.wroc.pl), Grant No. 268.

\section{APPENDIX A: NORMALIZATION}

The Dirac field of a 1/2-particle of mass $M$ and momentum $p$ is normalized so that

$$
\begin{gathered}
\bar{u}(p, s) u\left(p, s^{\prime}\right)=2 M \delta_{s s^{\prime}} \\
u(p, s) \bar{u}(p, s)=\frac{1}{2} \gamma_{5} \not(\not p+M),
\end{gathered}
$$

where $\not p \equiv p_{\mu} \gamma^{\mu}$.

One particle fermion/scalar state is normalized so that

$$
\left\langle\mathbf{p}^{\prime}, s^{\prime} \mid \mathbf{p}, s\right\rangle=2 E_{p}(2 \pi)^{3} \delta_{s s^{\prime}} \delta^{(3)}\left(\mathbf{p}-\mathbf{p}^{\prime}\right),
$$

where $|\mathbf{p}, s\rangle \equiv a^{\dagger}(\mathbf{p}, s)|0\rangle$, and $a^{\dagger}$ is the creation operator of the particle with momentum $\mathbf{p}$ and spin $s$.

\section{APPENDIX B: TRANSITION FORM FACTORS}

The vector nucleon form factors $F_{1}^{V}$ and $F_{2}^{V}$ are expressed in terms of the electromagnetic neutron, $F_{k}^{n}$, and proton, $F_{k}^{p}$, form factors, namely,

$$
F_{k}^{V}(q)=F_{k}^{p}(q)-F_{k}^{n}(q), \quad k=1,2 .
$$

We consider the same nucleon form factors as in [8]. 
The axial nucleon form factor reads

$$
G_{A}(q)=\frac{g_{A}}{\left(1-\frac{q^{2}}{M_{A}^{2}}\right)^{2}},
$$

where $M_{A}=1.00 \mathrm{GeV}$ and $g_{A}=1.26$.

\section{HNV model}

The vector $N \rightarrow \Delta(1232)$ transition form factors are given by [46]

$$
\begin{gathered}
C_{3}^{V}\left(Q^{2}\right)=\frac{C_{3}^{V}(0)}{1+A Q^{2}+B Q^{4}+C Q^{6}} \cdot\left(1+K_{1} Q^{2}\right), \\
C_{4}^{V}\left(Q^{2}\right)=-\frac{M}{W} C_{3}^{V}\left(Q^{2}\right) \cdot \frac{1+K_{2} Q^{2}}{1+K_{1} Q^{2}}, \\
C_{5}^{V}\left(Q^{2}\right)=\frac{C_{5}^{V}(0)}{\left(1+D \frac{Q^{2}}{M_{V}^{2}}\right)^{2}},
\end{gathered}
$$

where $M_{V}=0.84 \mathrm{GeV}$, and parameters $K_{1}, K_{2}, A, B, C$, and $D$ are given in Table II.

The axial form factor $C_{5}^{A}\left(Q^{2}\right)$ has the form

$$
C_{5}^{A}\left(Q^{2}\right)=\frac{C_{5}^{A}(0)}{\left(1+\frac{Q^{2}}{M_{A \Delta}^{2}}\right)^{2}},
$$

where $M_{A \Delta}=0.85 \mathrm{GeV}$ and $C_{5}^{A}(0)=1.10$ as obtained in Ref. [46].

$F_{\rho}$ is given by [8]

$$
F_{\rho}(q)=\frac{1}{1-q^{2} / m_{\rho}^{2}},
$$

where $m_{\rho}=0.7758 \mathrm{GeV}$.

\section{FN model}

The $C_{3}^{V}$ vector form factor for the $N \rightarrow \Delta$ transition reads [56]
TABLE II. Parameters of the vector form factors for the $N \rightarrow \Delta$ transition.

\begin{tabular}{lccccccc}
\hline \hline$C_{3}^{V}(0)$ & $C_{5}^{V}(0)$ & $A$ & $B$ & $C$ & $D$ & $K_{1}$ & $K_{2}$ \\
\hline 2.10 & 0.63 & 4.73 & -0.39 & 5.59 & 1.00 & 0.13 & 1.68 \\
\hline \hline
\end{tabular}

$C_{3}^{V}\left(Q^{2}\right)=2.07 \exp \left(-3.15 \frac{\sqrt{Q^{2}}}{\mathrm{GeV}}\right) \sqrt{\left(1+9 \frac{\sqrt{Q^{2}}}{\mathrm{GeV}}\right)}$.

The axial form factor $C_{5}^{A}\left(Q^{2}\right)$ is parametrized by (B6) with parameters [11] $M_{A \Delta}=0.75 \mathrm{GeV}$ and $C_{5}^{A}(0)=1.18$. The pion form factor reads

$$
F_{\pi}(q)=\frac{1}{1-q^{2} /\left(0.47 \mathrm{GeV}^{2}\right)}
$$

\section{APPENDIX C: $\Delta(1232)$ RESONANCE WIDTHS}

In the HNV model, the $\Delta(1232)$ resonance width reads

$$
\begin{aligned}
\Gamma_{\Delta}^{\mathrm{HNV}}(s)= & \frac{1}{6 \pi}\left(\frac{f^{*}}{m_{\pi}}\right)^{2} \frac{M}{\sqrt{s}} \\
& \times\left[\frac{\lambda\left(s, M^{2}, m_{\pi}^{2}\right)}{2 \sqrt{s}}\right]^{3} \theta\left(\sqrt{s}-M-m_{\pi}\right),
\end{aligned}
$$

where

$$
\lambda(x, y, z)=x^{2}+y^{2}+z^{2}-2 x y-2 x z-2 y z
$$

In the FN model, the width takes a similar form:

$$
\begin{aligned}
\Gamma_{\Delta}^{\mathrm{FN}}(s)= & \frac{1}{6 \pi}\left(\frac{f^{*}}{m_{\pi}}\right)^{2} \frac{1}{(2 \sqrt{s})^{5}}\left[(\sqrt{s}+M)^{2}-m_{\pi}^{2}\right] \\
& \times\left[\lambda\left(s, M^{2}, m_{\pi}^{2}\right)\right]^{3} \theta\left(\sqrt{s}-M-m_{\pi}\right) .
\end{aligned}
$$

[1] C. H. L. Smith, Phys. Rep. 3, 261 (1972).

[2] A. A. Aguilar-Arevalo et al. (MiniBooNE Collaboration), Phys. Rev. Lett. 98, 231801 (2007).

[3] K. Abe et al. (T2K Collaboration), Nucl. Instrum. Methods Phys. Res., Sect. A 659, 106 (2011).

[4] J. Evans (MINOS Collaboration), Adv. High Energy Phys. 2013, 182537 (2013).
[5] D. S. Ayres et al. (NOvA Collaboration), arXiv:hep-ex/ 0503053.

[6] L. Aliaga et al. (MINERvA), Nucl. Instrum. Methods Phys. Res., Sect. A 743, 130 (2014).

[7] U. Mosel, Annu. Rev. Nucl. Part. Sci. 66, 171 (2016).

[8] E. Hernandez, J. Nieves, and M. Valverde, Phys. Rev. D 76, 033005 (2007). 
[9] S. L. Adler, Ann. Phys. (N.Y.) 50, 189 (1968).

[10] D. Rein and L. M. Sehgal, Ann. Phys. (N.Y.) 133, 79 (1981).

[11] G. L. Fogli and G. Nardulli, Nucl. Phys. B160, 116 (1979).

[12] D. Rein, Z. Phys. C 35, 43 (1987).

[13] E. Hernandez, J. Nieves, and M. Valverde, Phys. Lett. B 647, 452 (2007).

[14] S. X. Nakamura, H. Kamano, and T. Sato, Phys. Rev. D 92, 074024 (2015).

[15] B. D. Serot and X. Zhang, Phys. Rev. C 86, 015501 (2012).

[16] O. Lalakulich, T. Leitner, O. Buss, and U. Mosel, Phys. Rev. D 82, 093001 (2010).

[17] T. Leitner, O. Buss, L. Alvarez-Ruso, and U. Mosel, Phys. Rev. C 79, 034601 (2009).

[18] M. R. Alam, M. S. Athar, S. Chauhan, and S. K. Singh, Int. J. Mod. Phys. E 25, 1650010 (2016).

[19] C. Barbero, G. L. Castro, and A. Mariano, Phys. Lett. B 664, 70 (2008).

[20] R. Gonzalez-Jimenez, N. Jachowicz, K. Niewczas, J. Nys, V. Pandey, T. Van Cuyck, and N. Van Dessel, Phys. Rev. D 95, 113007 (2017).

[21] L. Alvarez-Ruso, Y. Hayato, and J. Nieves, New J. Phys. 16, 075015 (2014).

[22] G. M. Radecky, V. E. Barnes, D. D. Carmony, A. F. Garfinkel, M. Derrick, E. Fernandez, L. Hyman, G. Levman et al., Phys. Rev. D 26, 1161 (1982); 26, 3297(E) (1982).

[23] T. Kitagaki, H. Yuta, S. Tanaka, A. Yamaguchi, K. Abe et al., Phys. Rev. D 34, 2554 (1986).

[24] O. Altinok et al. (MINERvA Collaboration), Phys. Rev. D 96, 072003 (2017).

[25] A. I. Akhiezer and M. Rekalo, Dokl. Akad. Nauk Ser. Fiz. 180, 1081 (1968) [Sov. Phys. Dokl. 13, 572 (1968)].

[26] A. I. Akhiezer and M. Rekalo, Fiz. Elem. Chastits At. Yadra 4, 662 (1973) [Sov. J. Part. Nucl. 4, 277 (1974)].

[27] G. G. Ohlsen, Rep. Prog. Phys. 35, 717 (1972).

[28] R. G. Arnold, C. E. Carlson, and F. Gross, Phys. Rev. C 23, 363 (1981).

[29] T. W. Donnelly and A. S. Raskin, Ann. Phys. (N.Y.) 169, 247 (1986).

[30] A. Afanasev, P. G. Blunden, D. Hasell, and B. A. Raue, Prog. Part. Nucl. Phys. 95, 245 (2017).

[31] S. M. Bilenky and E. Christova, Phys. Part. Nucl. Lett. 10, 651 (2013).

[32] S. M. Bilenky and E. Christova, J. Phys. G 40, 075004 (2013).
[33] F. Akbar, M. S. Athar, A. Fatima, and S. K. Singh, Eur. Phys. J. A 53, 154 (2017).F. Akbar, M. Rafi Alam, M. Sajjad Athar, and S. K. Singh, Phys. Rev. D 94, 114031 (2016).

[34] K. S. Kuzmin, V. V. Lyubushkin, and V. A. Naumov, Mod. Phys. Lett. A 19, 2815 (2004); Phys. Part. Nucl. 35, S133 (2004).

[35] K. Hagiwara, K. Mawatari, and H. Yokoya, Nucl. Phys. B668, 364 (2003); B701, 405(E) (2004).

[36] K. S. Kuzmin, V. V. Lyubushkin, and V. A. Naumov, Nucl. Phys. B. Proc. Suppl. 139, 154 (2005).

[37] K. M. Graczyk, Nucl. Phys. A748, 313 (2005).

[38] N. Dombey, Rev. Mod. Phys. 41, 236 (1969).

[39] W. Greiner, Quantum Electrodynamics (Springer-Verlag, Berlin Heidelberg, 1992).

[40] L. C. Maximon and W. C. Parke, Phys. Rev. C 61, 045502 (2000).

[41] J. D. Walecka, Electron Scattering for Nuclear and Nucleon Structure (Cambridge University Press, Cambridge, 2005).

[42] L. Alvarez-Ruso, E. Hernandez, J. Nieves, and M. J. V. Vacas, Phys. Rev. D 93, 014016 (2016).

[43] E. Hernandez and J. Nieves, Phys. Rev. D 95, 053007 (2017).

[44] W. Rarita and J. Schwinger, Phys. Rev. 60, 61 (1941).

[45] H. Jones and M. Scadron, Ann. Phys. (N.Y.) 81, 1 (1973).

[46] K. M. Graczyk, J. Zmuda, and J. T. Sobczyk, Phys. Rev. D 90, 093001 (2014).

[47] P. A. Schreiner and F. Von Hippel, Nucl. Phys. B58, 333 (1973).

[48] K. M. Graczyk, Proc. Sci., EPS-HEP2009 (2009) 286 [arXiv:0909.5084].

[49] K. M. Graczyk and J. T. Sobczyk, Phys. Rev. D 77, 053001 (2008); 79, 079903(E) (2009).

[50] J. Zmuda, Ph.D. thesis, Institute of Theoretical Physics, University of Wrocław, Poland, 2013.

[51] J. J. de Swart, M.C. M. Rentmeester, and R. G. E. Timmermans, PiN Newslett. 13, 96 (1997).

[52] E. Hernandez, J. Nieves, M. Valverde, and M. J. V. Vacas, Phys. Rev. D 81, 085046 (2010).

[53] J.-J. Wu, T. Sato, and T. S. H. Lee, Phys. Rev. C 91, 035203 (2015).

[54] K. Abe et al. (T2K Collaboration), Phys. Rev. D 87, 012001 (2013); 87, 019902(E) (2013).

[55] J. A. M. Vermaseren, arXiv:math-ph/0010025.

[56] A. J. Dufner and Y.-S. Tsai, Phys. Rev. 168, 1801 (1968). 